\title{
Theoretical Analysis of the Entrainment-Mixing Process at Cloud Boundaries. Part II: Motion of Cloud Interface
}

\author{
MARK Pinsky AND AleXANDER KHAIN \\ Department of Atmospheric Sciences, Hebrew University of Jerusalem, Jerusalem, Israel
}

(Manuscript received 19 October 2018, in final form 11 February 2019)

\begin{abstract}
The entrainment-mixing process leads to formation of a cloud-environment interface zone, consisting of the cloud dilution zone, where the liquid water content decreases as the distance from the cloud interior increases, and a humid shell with higher humidity in comparison with the dry environment. The time evolution of the location and width of these zones is analyzed by solving the diffusion-evaporation equation for the open region in the vicinity of the cloud-dry air interface. Upon normalization, the problem is reduced to a one-parametric one, the governing parameter being the potential evaporation parameter $R<0$, which is proportional to the ratio of saturation deficit in the dry air to the available liquid water content in the cloud air. It is shown that the widths of the dilution zone and the humid shell increase with time. At $R<-1$, the interface between the dilution zone and the humid shell (i.e., the cloud edge) moves toward the cloud interior (i.e., the cloud dissipates). If $R>-1$, the cloud edge moves outward; that is, the mixing leads to an increase in the cloud volume. It is shown that the relative humidity remains high within most of the cloud dilution zone, and the effective radius changes only slightly, especially in growing clouds, where the effective radius changes by less than $10 \%$ within more than $80 \%$ of the cloud dilution zone. The strong decrease in the LWC is accompanied by a corresponding decrease in the droplet concentration. The study is illustrated by means of two examples of clouds: a small cumulus $(\mathrm{Cu})$ typical of the trade wind zone and a deep convective cloud typical of the monsoon period over India. It is shown that while the small $\mathrm{Cu}$ tends to dissipate, the deep convective cloud expands. The characteristic sizes of the dilution zone and the humid shell are several tens of meters for the small $\mathrm{Cu}$ and several hundred meters for the deep convective cloud.
\end{abstract}

\section{Introduction}

Entrainment and mixing at cloud edges lead to a quite complicated structure of the interface zone surrounding the cloud edge. According to in situ measurements (Gerber et al. 2008; Katzwinkel et al. 2014; Schmeissner et al. 2015; Bera et al. 2016a,b; Kumar et al. 2017), as well as to numerical simulations using the LagrangianEulerian model (LEM; Magaritz-Ronen et al. 2016; Khain et al. 2018), entrainment of dry environment air into a cloud and subsequent mixing lead to a decrease in liquid water content (LWC) within the dilution zone of width ranging from several tens to several hundreds of meters from the cloud edge. This in turn decreases the so-called adiabatic liquid water fraction, which is of minimum value at the vicinity of the cloud edge and monotonically increases inward through the cloud, up to

Corresponding author: Alexander Khain, alexander.khain@ mail.huji.ac.il values typical of the cloud interior. Such changes of the cloud microphysical structure occurring as the distance from the cloud edge to the cloud interior increases were also reported in direct numerical simulations (DNS) performed by Kumar et al. (2017), who compared the results of advanced DNS with high-resolution in situ measurements of microphysical parameters at the edges of convective clouds in India.

In situ measurements indicate the existence of subsiding shells around small cumulus $(\mathrm{Cu}$; Rodts et al. 2003; Heus and Jonker 2008; Katzwinkel et al. 2014; Park et al. 2017). It is often assumed that one of the mechanisms of formation of these subsiding shells is cooling caused by evaporation of droplets penetrating dry air. In some studies, the existence of a humid shell around $\mathrm{Cu}$ is suggested (Gerber et al. 2008; Lehmann et al. 2009; Bar-Or et al. 2012). A humid shell at the cloud edge is seen well in high-resolution in situ measurements (Katzwinkel et al. 2014; see Fig. 2 in that study). Gerber et al. (2008) and Lehmann et al. (2009) 


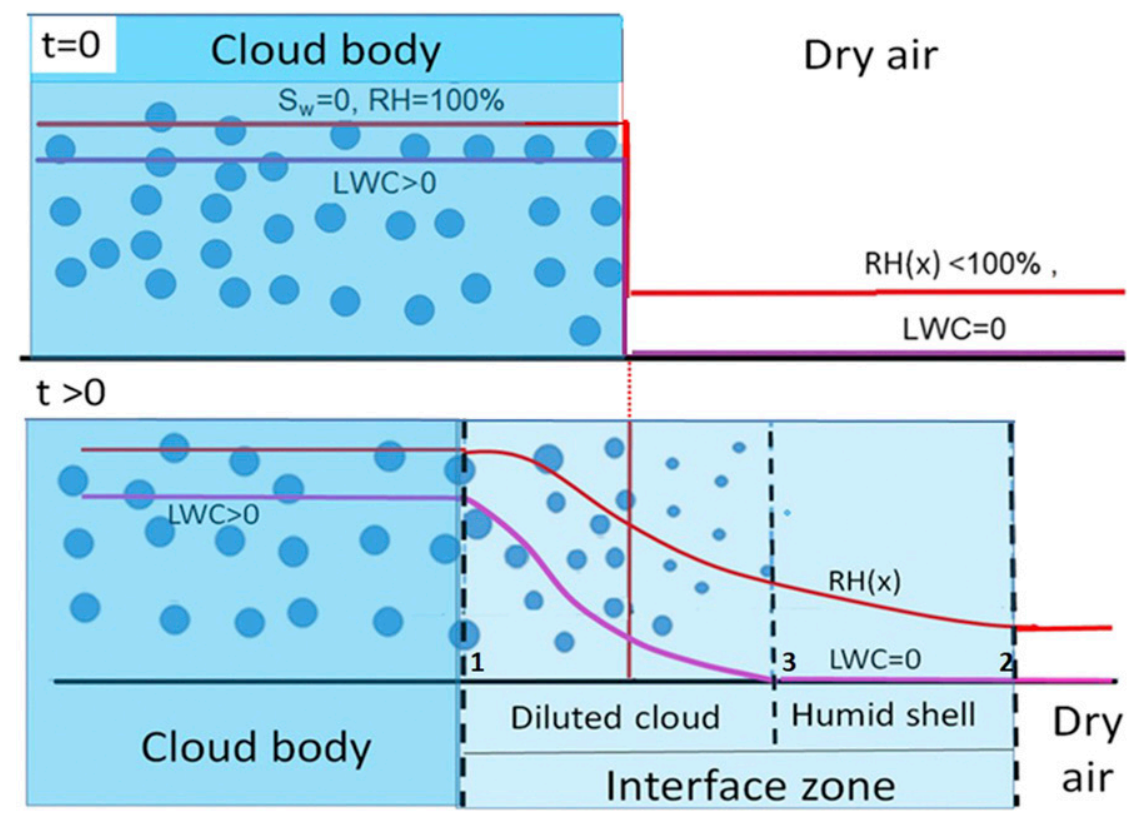

FIG. 1. Conceptual scheme of zones in the vicinity of the cloud-dry air interface (top) at $t=0$ and (bottom) for the developed interface zone. The initially sharp cloud-air interface is shown by red line in the top panel. Zone between vertical lines 1 and 3 is the dilution cloud zone. Zone between lines 3 and 2 is the humid shell [from Part I with changes].

refer to the humid shell around clouds to explain possible high humidity of entrained air. To the best of our knowledge, there is no quantitative description of the mechanism of formation of a humid shell within the interface zone and no evaluation of its width. In addition, no systematic experimental analysis of the interface-zone structure under different relative humidity (RH) of surrounding air has been performed. Understanding the mechanisms of formation of the humid shell, which can reach a few hundred meters in width, is highly important for proper calculation of cloud radiative fluxes that are strongly affected by water vapor remaining after droplet evaporation (Bar-Or et al. 2012). It is obvious that the effects of mixing on cloud microphysical structure and on cloud buoyancy depend on the humidity of the penetrating air (Plant and Yano 2015; Khain and Pinsky 2018; Khain et al. 2019).

Turbulent mixing at the cloud edge is a nonstationary process and may lead either to cloud dissipation (evaporation) when the cloud edge moves toward the cloud interior or to cloud growth when the edge shifts toward the dry environment. The entrainment-mixing processes affect cloud lifetime and cloud cover. The conditions leading to a particular scenario of cloud evolution are not known yet.

In this study, we present a theoretical analysis of the entrainment-mixing process in the vicinity of the clouddry air interface. The goals of the study are to provide a quantitative description of the evolution of the microphysical characteristics such as drop size distribution (DSD), its moments, and air humidity in the interface zone, and to find conditions fostering either cloud dissipation or cloud growth. In Pinsky and Khain (2018, hereafter Part I), we formulated the diffusionevaporation equation, describing the entrainmentmixing process at the cloud edge. We analyzed the formation of the interface zone structure and presented DSD as a function of time and space. Figure 1, presented also in Part I, shows a conceptual scheme of the cloud and its surrounding structure in the vicinity of the cloud edge.

We assume that gradients of all microphysical and thermodynamic quantities (in particular, of droplet concentration, LWC, and air humidity) at the cloud edge are initially sharp. The cloud is to the left of the edge, and the dry surrounding air is to the right. Entrainment and mixing lead to smoothing of these sharp gradients and to emergence of an interface zone with boundaries representing moving fronts marked in Fig. 1 by numbers 1 and 2 . Within this zone, entrainment and mixing affect the microphysical and thermodynamic structure. Outside the interface zone, changes of any cloud parameters and of parameters of the surrounding air are negligible. The interface zone consists of a zone where LWC changes (the dilution cloud zone) and a zone where the surrounding air humidity increases 
because of mixing at LWC close to zero (the humid shell). The boundaries of all zones move continuously, so the entrainment-mixing process is nonstationary and does not lead to a spatial homogenization typically assumed in multiple studies of mixing in clouds.

In part II of the study, we focus on the analysis of the motion of interfaces and time dependence of widths of the mixing zones. The changes of the droplet effective radius within the dilution zone, which are of practical interest, are also analyzed in the study. The behavior of effective radius is of special interest for two main reasons. First, the values of the effective radius affect the radiative cloud properties. Since the dilution zones may contribute significantly to the cloud cover, it is important to know the effective radius in the dilution zones to properly evaluate the radiative cloud properties. Second, changes of the effective radius are often used to establish the type of mixing (homogeneous vs inhomogeneous).

Time and spatial dependences of parameters of the interface zone, of the cloud dilution zone, and of the humid shell, as well as changes of the effective radius are analyzed both analytically and numerically by means of the diffusion-evaporation equation (Pinsky et al. 2016a,b; Part I). The analysis is performed under the following main simplifications:

- Mixing is caused by turbulence. The turbulent fluctuations in the inertial interval determine mutual diffusion of the inside-cloud and outside-cloud environments. There is no regular air advection; that is, both the horizontal and the vertical velocity are assumed to be zero.

- One-dimensional horizontal geometry is considered. The cloud area and the surrounding dry-air area are assumed to be infinitely large.

- The microphysical processes of droplet nucleation, collision-coalescence growth, and sedimentation are disregarded.

- The temperature changes caused by droplet evaporation during mixing are not taken into account.

\section{Brief description of the diffusion-evaporation model}

A detailed description of the model design is given in Part I of the study. In this paper, we briefly describe the main formulations employed in Part I. The derivation of the main equations can be found in appendix A. We consider mixing in the direction perpendicular to the vertical cloud-dry air interface. During mixing in the horizontal direction, the dry air with thermodynamic properties of the cloud surrounding and the cloudy air containing droplets interpenetrate. Herewith, different microphysical and thermodynamic quantities are mixed. This process of mutual diffusion is accompanied by droplet evaporation.

As shown in appendix A and in Part I, the model admits using dimensionless normalized variables. The equations represented in nondimensional form have solutions depending on the sole nondimensional parameter $R$ called potential evaporation parameter (PEP):

$$
R=\frac{S_{2}}{A_{2} q_{1}}<0
$$

where $S_{2}<0$ is supersaturation of undisturbed surrounding air, $q_{1}$ is the liquid water mixing ratio in undisturbed cloud and $A_{2}$ is a coefficient increasing as the air temperature decreases (see definition of $A_{2}$ in appendix B). The PEP is negative and proportional to the ratio of the amount of water vapor that should be added in order to saturate the unit volume of the surrounding air to the liquid water amount available for evaporation in the unit cloud volume. Since $A_{2}$ increases with height because of the temperature decrease, clouds with high LWC or penetrating to high levels are characterized by larger values of $R$. It is also assumed that cloud air is saturated; that is, $\mathrm{RH}_{1}=1$.

The list of nondimensional variables indicated with the upper tilde symbol is presented in Table 1 . These variables applied in the model are related to the following dimensional variables presented in the Table 1 : $x$ is the distance from the initial interface location, $t$ is the time, $q(x, t)$ is liquid water mixing ratio, $\Gamma(x, t)=S(x, t)+A_{2} q(x, t)$ is the conservative function, $S(x, t)$ is supersaturation, $\sigma=r^{2}$ is square of droplet radius $r$, and $g(x, t, \sigma)$ is distribution of droplets with respect to square radii. The initially given values listed in Table 1 are as follows: $K$ is the turbulent coefficient and $N_{1}, r_{1}$, and $\tau_{1}=\rho_{a} F /\left(4 \pi \rho_{w} A_{2} N_{1} r_{1}\right)$ are droplet concentration, droplet radius, and droplet relaxation time, respectively, in an undisturbed cloud. The notations of other values are given in appendix B.

The main equation of the diffusion-evaporation model is the equation for DSD $g(\sigma)$. Its nondimensional form is (see appendix A and Part I)

$$
\frac{\partial \tilde{g}(\tilde{x}, \tilde{t}, \tilde{\sigma})}{\partial \tilde{t}}=\frac{\partial^{2} \tilde{g}(\tilde{x}, \tilde{t}, \tilde{\sigma})}{\partial \tilde{x}^{2}}+\frac{2}{3}[\tilde{\Gamma}(\tilde{x}, \tilde{t})-\tilde{q}(\tilde{x}, \tilde{t})] \frac{\partial \tilde{g}(\tilde{x}, \tilde{t}, \tilde{\sigma})}{\partial \tilde{\sigma}} .
$$

The first term on the right-hand side of Eq. (2) describes changes in the DSD due to spatial diffusion along the $\tilde{x}$ axis, while the second term on the right-hand side 
TABLE 1. Main parameters of the model and their nondimensional forms. All nonnormalized values depend on the initially given values of $N_{1}, r_{1}, A_{2}, S_{2}$, and $K$.

\begin{tabular}{lccc}
\hline \hline \multicolumn{1}{c}{ Quantity } & Symbol & Nondimensional form & Range of normalized values \\
\hline Square of drop radius & $\sigma$ & $\tilde{\sigma}=\frac{\sigma}{r_{1}^{2}}$ & {$[0,1]$} \\
Droplet concentration & $N$ & $\tilde{N}=\frac{N}{N_{1}}$ & {$[0,1]$} \\
Liquid water mixing ratio & $q$ & $\tilde{q}=\frac{q}{q_{1}}$ & {$[0,1]$} \\
Distribution of square of drop radius & $g(\sigma)$ & $\tilde{g}(\tilde{\sigma})=\frac{r_{1}^{2}}{N_{1}} g(\sigma)$ & - \\
Conservative function & $\Gamma$ & $\tilde{\Gamma}=\frac{\Gamma}{A_{2} q_{1}}$ & {$[-\infty, 1]$} \\
Supersaturation & $S$ & $\tilde{S}=\frac{S}{A_{2} q_{1}}$ & {$[-\infty, 0]$} \\
Potential evaporation parameter & $R$ & $R=\frac{S_{2}}{A_{2} q_{1}}$ & {$[-\infty, 0]$} \\
Time & $t$ & $\tilde{t}=\frac{t}{\tau_{1}}$ & {$[0, \infty]$} \\
Distance & $x$ & $\tilde{x}=\frac{x}{\sqrt{K \tau_{1}}}$ & {$[-\infty, \infty]$} \\
\hline
\end{tabular}

describes changes in the DSD due to droplet evaporation. Equation (2) is solved using normalized boundary conditions of the Dirichlet type,

$$
\tilde{g}(-\infty, \tilde{t}, \tilde{\sigma})=\delta(\tilde{\sigma}-1) ; \quad \tilde{g}(\infty, \tilde{t}, \tilde{\sigma})=0,
$$

and the normalized initial condition of step type (Fig. 1)

$$
\tilde{g}(\tilde{x}, 0, \tilde{\sigma})=\left\{\begin{array}{ccc}
\delta(\tilde{\sigma}-1) & \text { if } & \tilde{x} \leq 0 \\
0 & \text { if } & \tilde{x}>0 .
\end{array}\right.
$$

Conditions (3) and (4) correspond to the initial location of the interface at $\tilde{x}=0$. The infinite cloud interior is located to the left of the interface; the infinite zone of surrounding dry air is located to the right of the interface (Fig. 1). Conditions (3) and (4) also mean that we assume monodisperse DSD in an undisturbed cloud.

To close Eq. (2), the normalized liquid water mixing ratio $\tilde{q}(\tilde{x}, \tilde{t})$ is calculated by integration of normalized DSD as

$$
\tilde{q}(\tilde{x}, \tilde{t})=\int_{0}^{\infty} \tilde{\sigma}^{3 / 2} \tilde{g}(\tilde{x}, \tilde{t}, \tilde{\sigma}) d \tilde{\sigma} .
$$

Then the normalized conservative function $\tilde{\Gamma}(\tilde{x}, \tilde{t})$,

$$
\tilde{\Gamma}(\tilde{x}, \tilde{t})=\tilde{S}(\tilde{x}, \tilde{t})+\tilde{q}(\tilde{x}, \tilde{t}),
$$

is calculated from the conventional equation of diffusion,

$$
\frac{\partial \tilde{\Gamma}(\tilde{x}, \tilde{t})}{\partial \tilde{t}}=\frac{\partial^{2} \tilde{\Gamma}(\tilde{x}, \tilde{t})}{\partial \tilde{x}^{2}}
$$

which is also solved with the Dirichlet boundary conditions

$$
\tilde{\Gamma}(-\infty, \tilde{t})=1 ; \quad \tilde{\Gamma}(\infty, \tilde{t})=R
$$

and the step initial conditions

$$
\tilde{\Gamma}(\tilde{x}, 0)=\left\{\begin{array}{lll}
1 & \text { if } & \tilde{x} \leq 0 \\
R & \text { if } & \tilde{x}>0 .
\end{array}\right.
$$

Therefore, system (2)-(9) determining the nonstationary solution is closed. It is seen from Eqs. (2)-(9) that the solution depends on the sole nondimensional parameter $R$. The solution of Eq. (2) allows calculation of profiles of different microphysical quantities at different time instances such as $\tilde{q}(\tilde{x}, \tilde{t}), \tilde{S}(\tilde{x}, \tilde{t})$, as well as the effective radius and other DSD moments, and thus to describe the evolution of the cloud dilution zone and the humid shell, whereas the solution of Eq. (7), $\tilde{\Gamma}(\tilde{x}, \tilde{t})$, determines the characteristics of the interface zone.

\section{Dynamics of the interface zone}

The zone where $\tilde{\Gamma}(\tilde{x}, \tilde{t})$ changes is the interface zone shown in Fig. 1. Outside this zone, changes of any microphysical and thermodynamic variables are negligible. Spatial-time changes of quantity $\tilde{\Gamma}(\tilde{x}, \tilde{t})$ can be obtained analytically using Eqs. (7)-(9). The analytical solution is (see Part I)

$$
\tilde{\Gamma}(\tilde{x}, \tilde{t})=\frac{1}{2}\left[(1+R)-(1-R) \operatorname{erf} \frac{\tilde{x}}{2 \sqrt{\tilde{t}}}\right],
$$




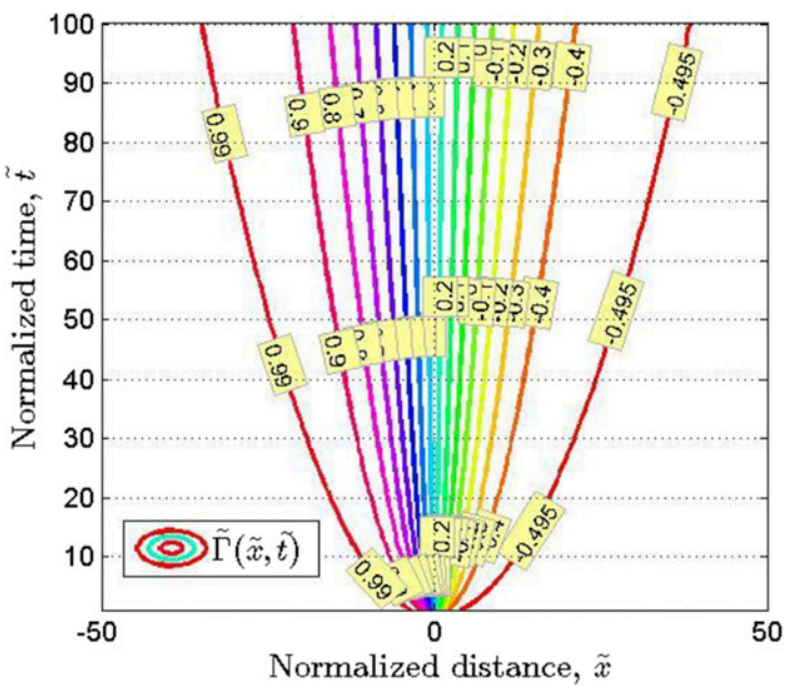

FIG. 2. Spatial-temporal changes of the conservative function $\tilde{\Gamma}(\tilde{x}, \tilde{t})$ with $R=-0.5$

where $\operatorname{erf}(z)$ is the error function. Since $\operatorname{erf}(-\infty)=-1$ and $\operatorname{erf}(\infty)=1$, this solution has two asymptotes:

$$
\tilde{\Gamma}(-\infty, \tilde{t})=1 ; \quad \tilde{\Gamma}(\infty, \tilde{t})=R<0 .
$$

The function $\tilde{\Gamma}(\tilde{x}, \tilde{t})$ becomes antisymmetric with respect to $x$ at $R=-1$.

Spatial-temporal changes of the conservative function $\tilde{\Gamma}(\tilde{x}, \tilde{t})$ are shown in Fig. 2 . One can see that expansion of $\tilde{\Gamma}(\tilde{x}, \tilde{t})$ increases with time. The left and right boundaries of the expansion correspond to asymptotes $\tilde{\Gamma}(-\infty, \tilde{t})=1$ and $\tilde{\Gamma}(\infty, \tilde{t})=R=-0.5$, respectively.

We define the location of boundaries of the interface zone in such a way that beyond them the changes of the parameters determining the zone do not exceed $\delta=1 \%$ of their asymptotic values. Outside the interface zone, the changes of the corresponding values are negligible. The shifts of the corresponding contours from the initial interface location over time are considered as movements of two fronts related to the interface zone expansion. The time shifts of the contours $\tilde{\Gamma}(\tilde{x}, \tilde{t})=1-\delta=0.99$ and $\tilde{\Gamma}(\tilde{x}, \tilde{t})=R \times(1-\delta)=-0.495$, seen in Fig. 2, correspond to movement of the two fronts related to interface zone expansion: dry air moves toward the cloud interior (to the left of this contour, the cloud is actually undisturbed), while the cloudy air moves toward the environment air (to the right of this contour, the surrounding air is actually undisturbed). The normalized speed of the fronts movement depends on the sole nondimensional parameter $R$.

Equation (10) allows analytical calculation of the front locations and their velocities. The coordinate of the left front $X_{\Gamma}^{l}(\tilde{t})$ can be calculated from the equality $\tilde{\Gamma}\left[X_{\Gamma}^{l}(\tilde{t}), \tilde{t}\right]=1-\delta$. Equation (10) leads to

$$
\tilde{\Gamma}\left[X_{\Gamma}^{l}(\tilde{t}), \tilde{t}\right]=\frac{1}{2}\left[(1+R)-(1-R) \operatorname{erf} \frac{X_{\Gamma}^{l}}{2 \sqrt{\hat{t}}}\right]=1-\delta
$$

and then to the equation for the left-front location

$$
X_{\Gamma}^{l}(\tilde{t})=2 \sqrt{\tilde{t}} \vartheta_{\Gamma}^{l}(R)=2 \sqrt{\tilde{t}} \operatorname{erf}^{-1}\left(\frac{2 \delta}{1-R}-1\right),
$$

where $\operatorname{erf}^{-1}(z)$ is the inverse error function and $\vartheta_{\Gamma}^{l}(R)=\operatorname{erf}^{-1}[2 \delta /(1-R)-1]$. It is seen from Eq. (13) that $X_{\Gamma}^{l}(\tilde{t})<0$ since $\delta$ is a small value. The speed of the left front propagation can be calculated by differentiation of Eq. (13) with respect to $\tilde{t}$ :

$$
V_{\Gamma}^{l}(\tilde{t})=\frac{d X_{\Gamma}^{l}(\tilde{t})}{d \tilde{t}}=\tilde{t}^{-1 / 2} \vartheta_{\Gamma}^{l}(R) .
$$

In the same way, we obtain the right front location $X_{\Gamma}^{r}(\tilde{t})$ from the equality $\tilde{\Gamma}(\tilde{x}, \tilde{t})=R \times(1-\delta)$ as

$$
X_{\Gamma}^{r}(\tilde{t})=2 \sqrt{\tilde{t}} \vartheta_{\Gamma}^{r}(R)=2 \sqrt{\tilde{t}} \operatorname{erf}^{-1}\left(1+\frac{2 R \delta}{1-R}\right),
$$

and the velocity of this front $V_{\Gamma}^{r}(\tilde{t})$ is

$$
V_{\Gamma}^{r}(\tilde{t})=\frac{d X_{\Gamma}^{r}(\tilde{t})}{d \tilde{t}}=\tilde{t}^{-1 / 2} \vartheta_{\Gamma}^{r}(R) .
$$

Here, $\vartheta_{\Gamma}^{r}(R)=\operatorname{erf}^{-1}[1+2 R \delta /(1-R)]$. Since $X_{\Gamma}^{r}(\tilde{t})>0$ and $V_{\Gamma}^{r}(\tilde{t})>0$, the right front moves to the right.

From Eqs. (13)-(16), it is also possible to find the width $\Delta X_{\Gamma}(\tilde{t})$ of interface zone and the rate of the interface zone expansion, $V_{\Gamma}(\tilde{t})$ :

$$
\begin{aligned}
\Delta X_{\Gamma}(\tilde{t}) & =X_{\Gamma}^{r}(\tilde{t})-X_{\Gamma}^{l}(\tilde{t})=2 \sqrt{\tilde{t}}\left[\vartheta_{\Gamma}^{r}(R)-\vartheta_{\Gamma}^{l}(R)\right] \\
& =2 \sqrt{\tilde{t}} \Delta \vartheta_{\Gamma}(R), \\
V_{\Gamma}(\tilde{t}) & =\tilde{t}^{-1 / 2} \Delta \vartheta_{\Gamma}(R),
\end{aligned}
$$

where $\Delta \vartheta_{\Gamma}(R)=\operatorname{erf}^{-1}[1+2 R \delta /(1-R)]-\operatorname{erf}^{-1}[2 \delta /(1-$ $R)-1]$. Note that all the microphysical and thermodynamic quantities can vary only within the interface zone $\left[X_{\Gamma}^{l}(\tilde{t}) \quad X_{\Gamma}^{r}(\tilde{t})\right]$.

All the velocities are inversely proportional to the square root of time and depend on the sole parameter $R$. In general case, the shifts of the left and the right fronts with respect to their initial location, as well as the front velocities are different because of nonsymmetricity of the profile $\tilde{\Gamma}(\tilde{x}, 0)$. Equations (13)-(18) show that being oppositely directed, the speeds of the interface zone boundaries become equal at each time 


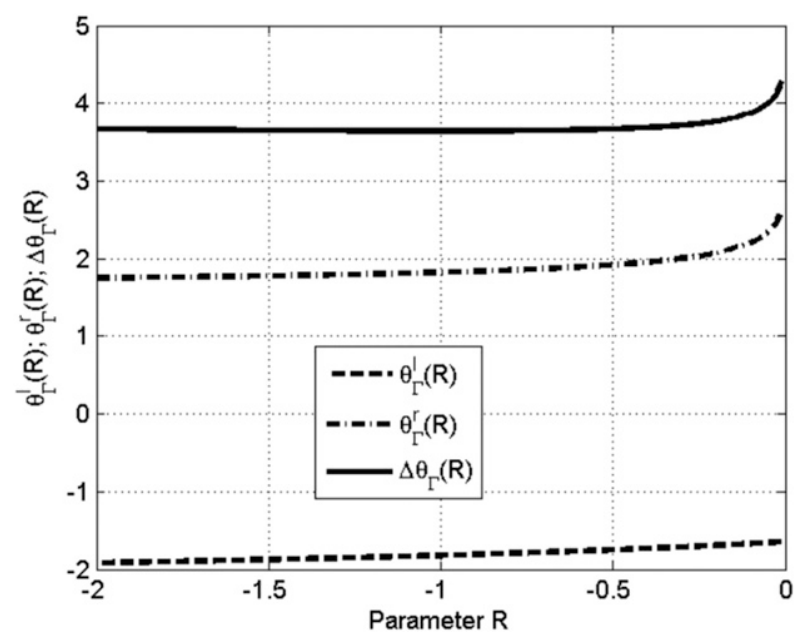

FIG. 3. Dependences $\vartheta_{\Gamma}^{l}(R), \vartheta_{\Gamma}^{r}(R)$, and $\Delta \vartheta_{\Gamma}(R)$.

instance when $R=-1$; that is, the expansion of the zone becomes symmetric. It means that at $R=-1$, the boundaries of the interface zone are symmetrically located with respect to $\tilde{x}=0$ at each time instance. The interface zone expands with time and its width increases as $\tilde{t}^{1 / 2}$. The rate of expansion decreases with the time as $\tilde{t}^{-1 / 2}$. This dependence represents the standard diffusion law for conservative quantities (Monin and Yaglom 1975).

Figure 3 shows dependences $\vartheta_{\Gamma}^{l}(R), \vartheta_{\Gamma}^{r}(R)$, and $\Delta \vartheta_{\Gamma}(R)$ at $\delta=0.01$. The dependences are weak except the zone near point $R=0$, that is, when either $\mathrm{RH}$ of the environment is close to $100 \%$ and/or the LWC in the cloud is large. At $R \rightarrow 0, \vartheta_{\Gamma}^{l} \rightarrow \operatorname{erf}^{-1}(2 \delta-1)=-1.645$, whereas $\vartheta_{\Gamma}^{r} \rightarrow \operatorname{erf}^{-1}(1+2 R \delta) \rightarrow \infty$, so the interface zone quickly expands because of the rapidly moving right front.

Figures $4 \mathrm{a}$ and $4 \mathrm{~b}$ illustrate the interface zone expansion. Generally, we can see a weak influence of parameter $R$ on the expansion of the interface zone formed by the conservative quantity $\tilde{\Gamma}(\tilde{x}, \tilde{t})$. Dependences $\Delta X_{\Gamma}(\tilde{t})$ and $V_{\Gamma}(\tilde{t})$ were calculated using Eqs. (17) and (18) for different values of parameter $R$. According to these equations, the interface zone expands with time and its width increases as $\tilde{t}^{1 / 2}$ (Fig. 4a). The rate of the expansion decreases with the time as $\tilde{t}^{-1 / 2}$ (Fig. 4b).

Since $\tilde{\Gamma}(\tilde{x}, \tilde{t})$ is a conservative value independent of phase transitions and is determined by diffusion only, the expansion of the interface zone is almost symmetric. Its dependence on $R$ is very weak and is related solely to the asymmetry of the initial profile of $\tilde{\Gamma}(\tilde{x}, 0)$.

\section{Dynamics of the cloud dilution zone and the humid shell}

Along with the interface zone, we consider the cloud dilution zone with boundaries $X_{q}^{l}(\tilde{t}), X_{q}^{r}(\tilde{t})$, within which all changes of LWC take place. We define the location of the left and the right boundaries of the dilution zone using the same criterion as that used to define the boundaries of the interface zone; namely, at $\tilde{x}=X_{q}(\tilde{t})$, the relative deviation from the asymptotic values at $\tilde{x}= \pm \infty$ is equal to $1 \%(\delta=0.01)$. Thus, $X_{q}^{l}(\tilde{t})$ actually coincides with $X_{\Gamma}^{l}(\tilde{t})$. One can also define the cloud dilution zone using the droplet concentration profile. As regards the humid shell, we assume that its left boundary $X_{S}^{l}(\tilde{t})$ coincides with the right boundary of the
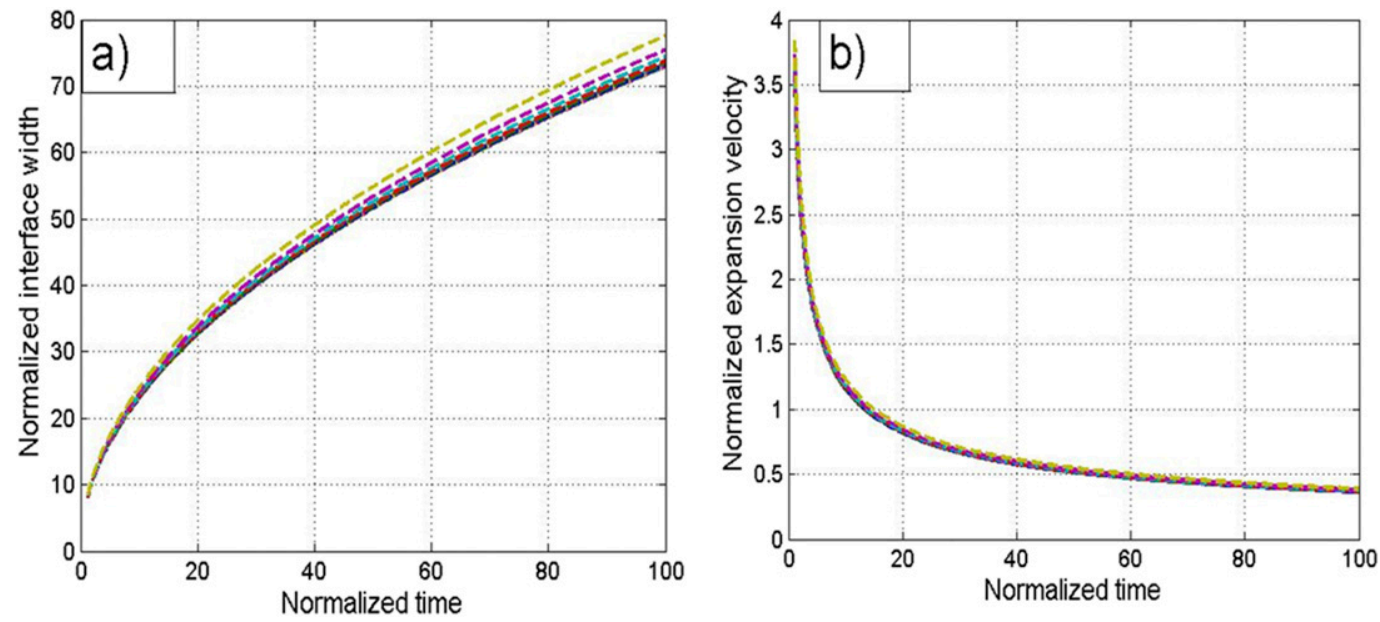

FIG. 4. Dependence of (a) the normalized interface zone width and (b) the normalized expansion velocity of the interface zone on the normalized time at different values of $R$. Parameter $R$ varies within the range of from -2 to -0.1 with an increment of 0.1 . The yellow curve corresponds to $R=-0.1$. 

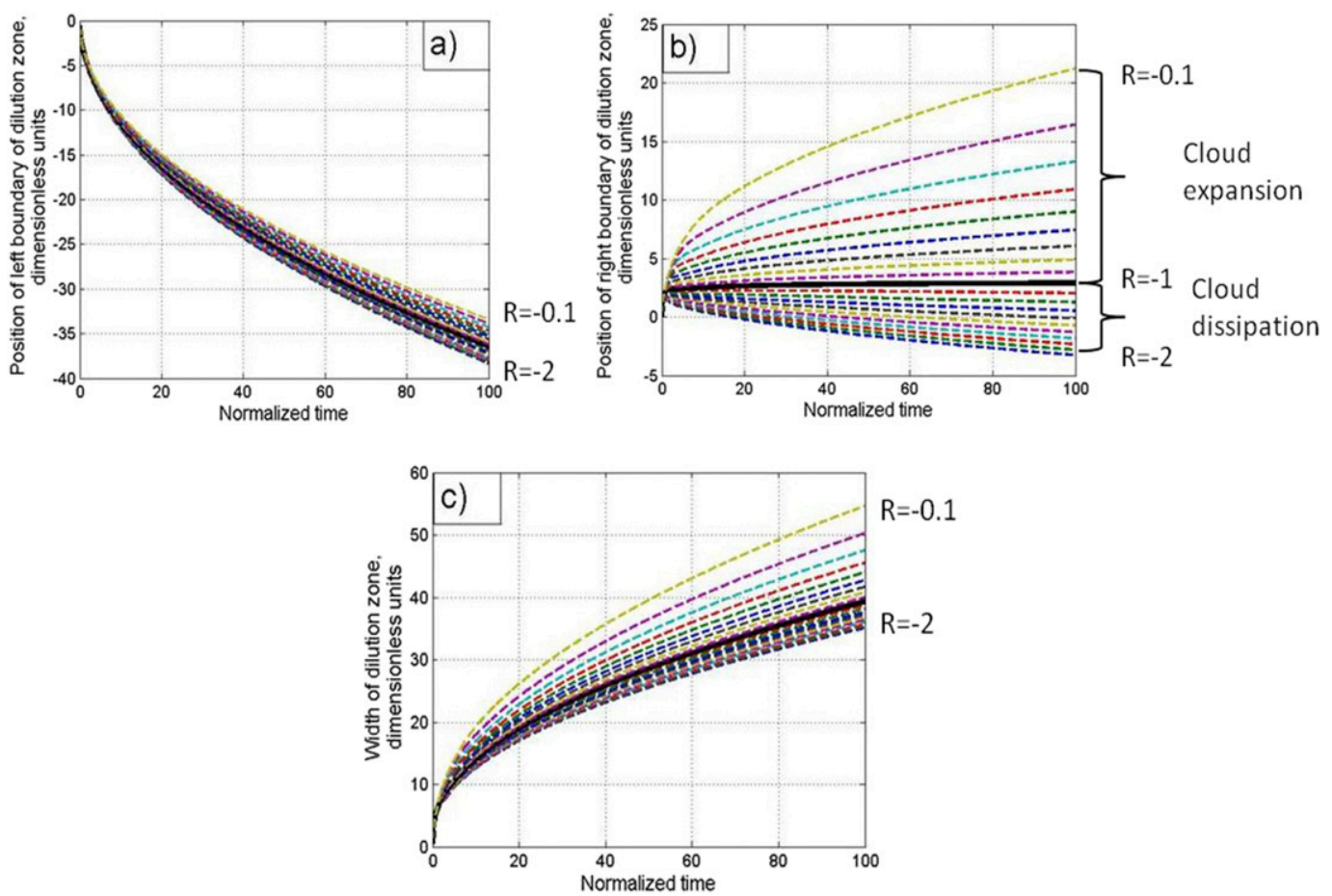

FIG. 5. Dependence of (a) $X_{q}^{l}(\tilde{t})$, (b) $X_{q}^{r}(\tilde{t})$, and (c) the normalized cloud dilution zone width on the normalized time at different values of $R$. Parameter $R$ varies within the range from -2 to -0.1 with an increment of 0.1 . Yellow curve corresponds to $R=-0.1$. Thick black line corresponds to $R=-1$, which separates the regimes of cloud expansion and cloud dissipation.

dilution zone $X_{q}^{r}(\tilde{t})$. The location of the right boundary of the humid shell $X_{S}^{r}(\tilde{t})$ is defined using the criterion of relative deviation $\delta=0.01(1 \%)$ from the asymptotic value of supersaturation at $\tilde{x}=\infty$.

To calculate the time evolution of the zones and their boundaries, we numerically solve the diffusionevaporation equation [Eq. (2)] for normalized DSD $\tilde{g}(\tilde{x}, \tilde{t}, \tilde{\sigma})$ using MATLAB solver pdepe and applying the initial condition [Eq. (4)] and the Dirichlet boundary conditions [Eq. (3)]. The calculations were carried out for parameter $R$ varying within the range from -2 up to -0.1 with increment of 0.1 . The results of the calculations are illustrated and analyzed below.

Figure 5 shows time changes of $X_{q}^{l}(\tilde{t}), X_{q}^{r}(\tilde{t})$, and the dilution zone width at different values of $R$. Figure 5a demonstrates that the left boundary of the cloud dilution zone always moves toward the cloud interior (because this boundary actually coincides with the left boundary of the interface zone), indicating that mixing with the environment always leads to some decrease in LWC and in droplet concentration inside the cloud. However, the direction of the motion of the right boundary $X_{q}^{r}(\widetilde{t})$ (Fig. 5b) significantly depends on the value of parameter $R$. The value $R=-1$ separates two regimes. Within the first regime at $R>-1$, the right boundary moves to the right toward the dry environment (the left and the right boundaries of the dilution zone move in different directions). It means that diffusion of wet cloud air and droplets causes an increase of the cloud volume. The higher $R$, the higher the velocity of cloud expansion is. It means that the rate of cloud expansion increases with increasing LWC and the RH of the dry surrounding air and with decreasing temperature (via the increase of $A_{2}$ coefficient). All these factors lead to faster saturation of dry surrounding air, caused by diffusion of droplets and their evaporation (total or partial).

The second regime corresponds to $R<-1$ when the process of droplet evaporation does not lead to saturation of the dry surrounding air, and the right boundary moves toward the cloud interior. This behavior indicates that at $R<-1$ the cloud dissipates and evaporates with time. In case $R=-1$, the location of the right boundary quickly stabilizes and a peculiar balance between the cloud and the environment takes place. Figure $5 \mathrm{c}$ shows that the width of the cloud dilution zone increases at any $R$ since at $R<-1$ the left boundary moves faster than the right one. The strongest expansion of the dilution zone takes place at large $R$, when movements of the right and the left boundaries have opposite directions. 


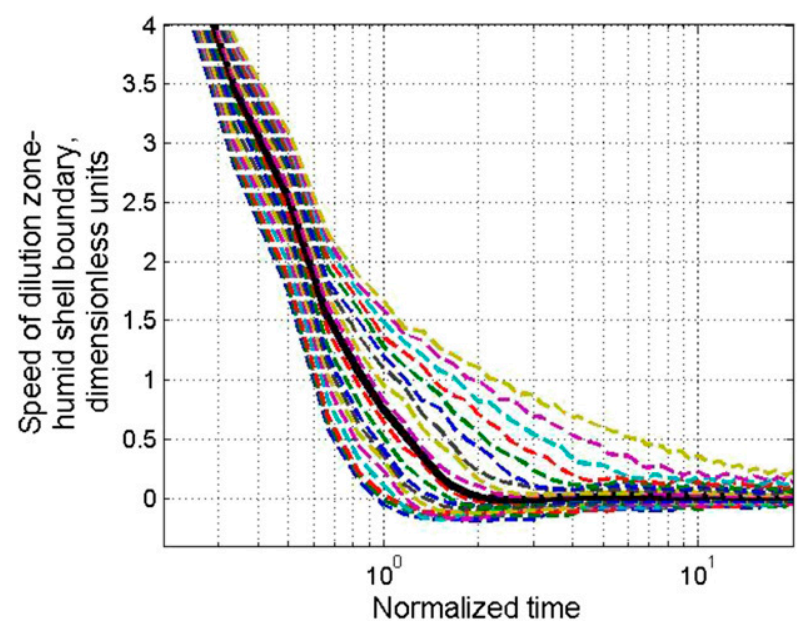

FIG. 6. Dependence of the velocity of the movement of the boundary between the cloud dilution zone and the humid shell on normalized time at different values of $R$. Parameter $R$ varies within the range from -2 to -0.1 with an increment of 0.1 . Yellow curve corresponds to $R=-0.1$. Thick black line corresponds to $R=-1$.

Figure 6 shows the dependence of velocity of the movement of the boundary between the cloud dilution zone and the humid shell on the normalized time. One can see that the velocity tends to zero over time at any $R$. However, the tendency is not the same for $R>-1$ and for $R<-1$. At $R<-1$ when the cloud dissipates, the right boundary of the cloud dilution zone (the left boundary of the humid shell) first moves to the right (positive velocity), and then changes direction. We attribute this effect to the fact that at $t=0$ the LWC gradient at the cloud edge is high and diffusion dominates causing the boundary to shift to the right toward the cloud environment. With time, the spatial gradient of LWC decreases and the role of droplet evaporation becomes dominating. This leads to the reverse in the direction of the cloud edge movement.

Figure 7 shows time changes of location of the humid shell boundaries $X_{S}^{l}(\tilde{t})$ and $X_{S}^{r}(\tilde{t})$ at different values of $R$. According to the definition, the $X_{S}^{l}(\tilde{t})$ coincides with $X_{q}^{r}(\tilde{t})$, so Figs. 5b and 7a are identical; $X_{S}^{r}(\tilde{t})$ actually also coincides with $X_{\Gamma}^{r}(\tilde{t})$. The right boundary of the humid shell moves toward the cloud environment at all values of $R$ (Fig. 7b). Its velocity is determined by turbulent diffusion of humidity. The highest velocity of the right boundary is reached at highest $R$, likely because high $R$ corresponds to higher gradients of humidity in the interface zone. Figure $7 \mathrm{c}$ shows that the normalized width of the humid shell is the largest in a dissipating cloud, when the velocities of the boundaries of the humid shell have opposite directions.
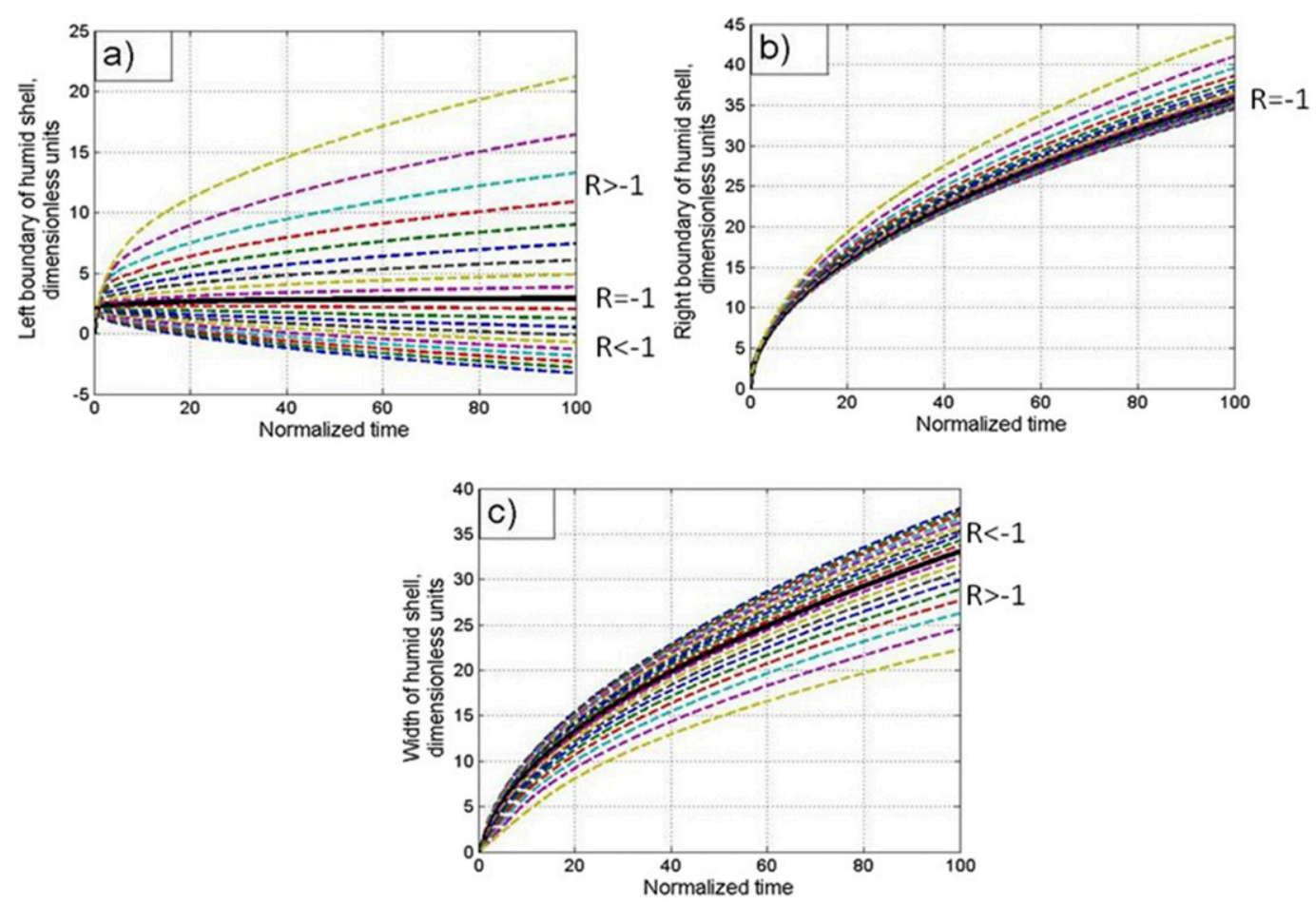

FIG. 7. (a) Dependence of $X_{S}^{l}(\tilde{t})$, (b) $X_{S}^{r}(\tilde{t})$, and (c) the normalized width of the humid shell on the normalized time at different values of $R$. Parameter $R$ varies within the range from -2 to -0.1 with an increment of 0.1 . Yellow curve corresponds to $R=-0.1$. Thick black line corresponds to $R=-1$. 


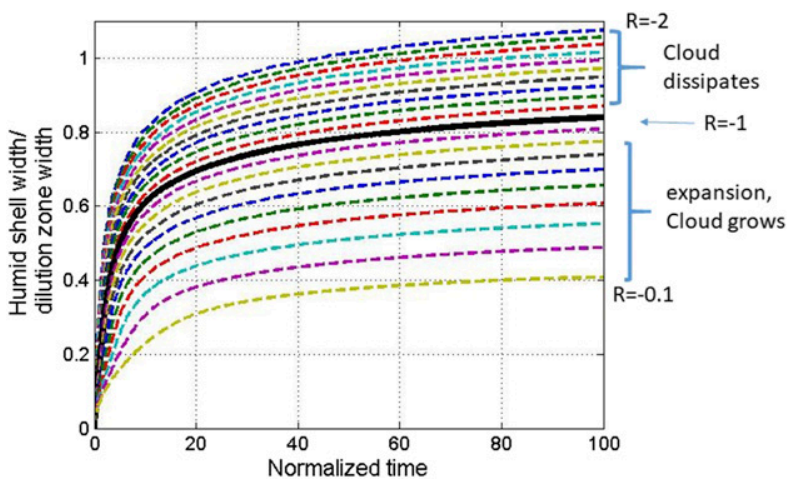

FIG. 8. Dependence of the humid shell/the dilution zone width ratio on the normalized time at different values of $R$. Parameter $R$ varies within the range from -2 to -0.1 with an increment of 0.1 . Yellow curve corresponds to $R=-0.1$. Thick black line corresponds to $R=-1$.

Figure 8 shows the dependence of humid shell/the dilution zone width ratio on the normalized time at different values of $R$. This ratio can be important when visible cloud sizes differ from sizes determined on the base of LWC. Over short time periods, the width of the humid shell is substantially smaller than that of the dilution zone, which forms first, while the humid shell forms with a delay as droplets moving from the cloud interior outside totally evaporate. Over long time periods, the humid shell/dilution zone width ratio tends toward constant values depending on $R$. Typically, the humid shell is narrower than the cloud dilution zone, with the exception of rapidly dissipating clouds (characterized by very small values of $R$ ). In such clouds, the left boundary of the humid shell (the right boundary of the dilution zone) shifts toward the cloud interior, while the right boundary of the humid shell moves outward of the cloud toward the environment. Within the wide range of $R$ values, the widths of the dilution zone and of the humid shall are of the same order.

In this study, we do not consider the spatial dependences of the normalized values of LWC, of droplet concentration and of supersaturation within the interface zone, since these dependences were discussed in Part I. It was found that $\mathrm{RH}$ within the cloud dilution zone is high and remains close to $100 \%$ within the larger fraction of the zone. $\mathrm{RH}$ decreases only within the region close to the right boundary of cloud dilution zone. This is explained by the fact that droplet evaporation supports high RH in this zone. Droplet concentration decreases within the dilution zone nearly proportionally to the decrease in LWC. Such proportionality breaks only in the vicinity of the cloud edge (the right boundary of the cloud dilution zone).

Cloud radiative properties depend to a large extent on the value of the effective droplet radius. In multiple

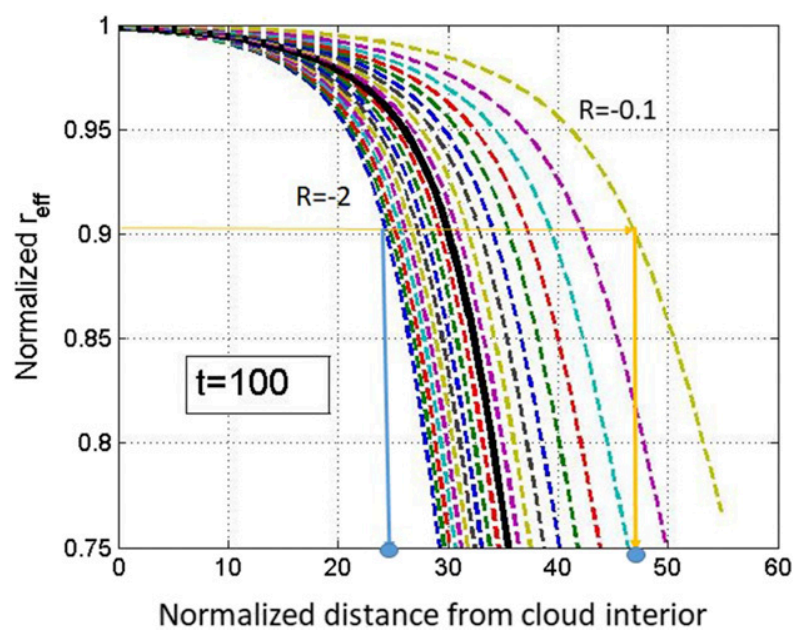

FIG. 9. Dependence of the normalized effective radius on the normalized distance from the cloud interior at different values of $R$ at normalized time $\tilde{t}=100$. Parameter $R$ varies within the range from -2 to -0.1 with an increment of 0.1 . Yellow dashed curve corresponds to $R=-0.1$. Thick black line corresponds to $R=-1$. Horizontal yellow line shows the normalized effective radius equal to 0.9 .

studies dedicated to effects of the entrainment-mixing process, the type of mixing is determined based on the dependences of the effective radius on LWC or on the droplet number concentration (e.g., Gerber 2000; Gerber et al. 2008; Freud et al. 2011; Pinsky et al. 2016b). As was shown in Part I, the relative humidity remains high within the dilution zone, and the effective radius changes only slightly within a larger fraction of the dilution zone, so that a strong decrease in LWC is accompanied by the corresponding decrease in the droplet concentration. Figure 9 shows the dependence of the normalized effective radius on the normalized distance from the cloud interior at different values of $R$ at normalized time $\tilde{t}=100$. At $R=-2$, the dilution zone width is equal to 35 nondimensional units, and the effective radius exceeds $90 \%$ of its maximum value at normalized distances below 25 units from the cloud interior $(90 \%$ of the dilution zone width). At $R=-0.1$, the dilution zone width is equal to 52 nondimensional units, and the effective radius exceeds $90 \%$ of its maximum value at normalized distances below 47 units from the cloud interior $(70 \%$ of the dilution zone width). Accordingly, a substantial decrease in the effective radius takes place within $10 \%$ of dilution zone in a growing cloud (at $R=-0.1$ ) and within $30 \%$ of the dilution zone in an evaporating cloud (at $R=-2$ ). Small changes of the effective radius within the dilution zone were observed during high-frequency in situ measurements in small $\mathrm{Cu}$ (Schmeissner et al. 2015). The profile of the characteristic radius shown in Fig. 9 is quite similar to that 
TABLE 2. Thermodynamic and microphysics parameters of clouds at cloud edges.

\begin{tabular}{|c|c|c|c|c|}
\hline Cloud parameter & Notation & Units & Cloud 1 & Cloud 2 \\
\hline Turbulent dissipation rate & $\varepsilon$ & $\mathrm{cm}^{2} \mathrm{~s}^{-3}$ & 10 & 200 \\
\hline External turbulent scale & $L$ & $\mathrm{~m}$ & 20 & 199 \\
\hline Droplet radius & $r_{1}$ & $\mu \mathrm{m}$ & 7.8 & 10 \\
\hline Droplet concentration & $N_{1}$ & $\mathrm{~cm}^{-3}$ & 200 & 400 \\
\hline LWC & $q_{1}$ & $\mathrm{~g} \mathrm{~kg}^{-1}$ & 0.4 & 1.7 \\
\hline Temperature & $T$ & ${ }^{\circ} \mathrm{C}$ & 20 & 2 \\
\hline Relative humidity of surrounding air & RH & $\%$ & 60 & 60 \\
\hline Turbulent coefficient & $K=0.2 \varepsilon^{1 / 3} L^{4 / 3}$ & $\mathrm{~m}^{2} \mathrm{~s}^{-1}$ & 1.1 & 63 \\
\hline Droplet relaxation time & $\tau_{1}=\frac{\rho_{a} F}{4 \pi \rho_{w} A_{2} N_{1} r_{1}}$ & $\mathrm{~s}$ & 1.6 & 0.72 \\
\hline Spatial scale & $\sqrt{K \tau_{1}}$ & $\mathrm{~m}$ & 1.3 & 6.8 \\
\hline Potential evaporation parameter & $R$ & - & -4.0 & -0.56 \\
\hline
\end{tabular}

measured by Kumar et al. (2017) at the edge of a growing convective cloud.

\section{Examples of evolution of zones in real clouds}

We illustrate the evolution of cloud structure caused by mixing at its lateral boundaries using as examples two clouds analyzed in Part I. Table 2 shows the initial thermodynamic and microphysical parameters of the clouds. Parameters of cloud 1 correspond to small trade wind $\mathrm{Cu}$ observed by Katzwinkel et al. (2014), while parameters of cloud 2 are suitable for deep convective clouds observed during monsoons in India, similar to those described by Kumar et al. (2017). Cloud 1 and cloud 2 are characterized by $R=-4<-1$ and $R=-0.56>-1$, respectively, so with time, cloud 1 should dissipate, whereas cloud 2 should grow. In both examples, the ratio between cloud parameters is such that the characteristic mixing time is much larger than the droplet relaxation time, so the mixing meets the description of the extremely inhomogeneous type. We believe that this is typical for clouds of different types. [The corresponding evaluations can be found in Pinsky et al. (2016a,b).] However, smallest trade wind clouds having the external turbulent scale on the order of several meters and a large turbulent dissipation rate may exist under certain conditions. In such clouds, the mixing scenario can be closer to the homogeneous type.

The development of the interface zone, the cloud dilution zone, and the humid shell in the two clouds described by Table 2 are shown in Fig. 10. One can see that in the deep convective cloud (right column), the interface zone and all the subzones within it rapidly grow and exceed several hundred meters right after the mixing starts. These values agree well with in situ observations presented by Bera et al. (2016a,b) and Kumar et al. (2017). For instance, from Fig. 5 in Bera et al. (2016a) and Fig. 13 in Kumar et al. (2017), one can conclude that the time period needed for crossing the cloud dilution zone (the adiabatic fraction changes from 0.01 to $0.5-0.7 \mathrm{~g} \mathrm{~kg}^{-1}$ ) is equal to $5-6 \mathrm{~s}$. Taking into account that aircraft speed is about $100 \mathrm{~m} \mathrm{~s}^{-1}$, the width of the dilution zone in such clouds can be estimated as 500-600 m. The same evaluation can be done on the basis of Fig. 3 in Bera et al. (2016b). The width of cloud periphery with low adiabatic fraction is evaluated as about $15 \%-20 \%$ of the cloud half width. The total width of deep convective clouds in these measurements is about $4-5 \mathrm{~km}$, which again allows to estimate the depth of the dilution zone as several hundred meters.

Figure 10 also shows that the width of the interface zone and of the subzones of the small $\mathrm{Cu}$ reaches several tens of meters, which agrees with in situ measurements in small $\mathrm{Cu}$ in several field experiments (Gerber et al. 2008; Katzwinkel et al. 2014; Schmeissner et al. 2015). For instance, 1000-Hz-frequency measurements in small $\mathrm{Cu}$ (Fig. 11 in Gerber et al. 2008) show that LWC increases from zero (cloud edge) to its maximum value of $0.52 \mathrm{~g} \mathrm{~m}^{-3}$ at the distance of $20-25 \mathrm{~m}$ from the edge.

The difference in the rates of the zone expansion can be attributed first of all to the difference in the values of turbulent coefficients determining the rate of turbulent front propagation.

Figure 10c shows that the outer boundary of the dilution zone in small $\mathrm{Cu}$ shifts toward the cloud interior, which indicates cloud dissipation. During first $10 \mathrm{~min}$ of mixing, the internal boundary of the dilution zone shifts by $100 \mathrm{~m}$ into the cloud interior. It means that clouds with size of a few hundred meters dilute during this time period (including cloud core, which is the inner undiluted part of the cloud). Thus, entrainmentmixing can substantially affect the lifetime of small $\mathrm{Cu}$. Figure $10 \mathrm{~d}$ shows that the outer boundary of the 

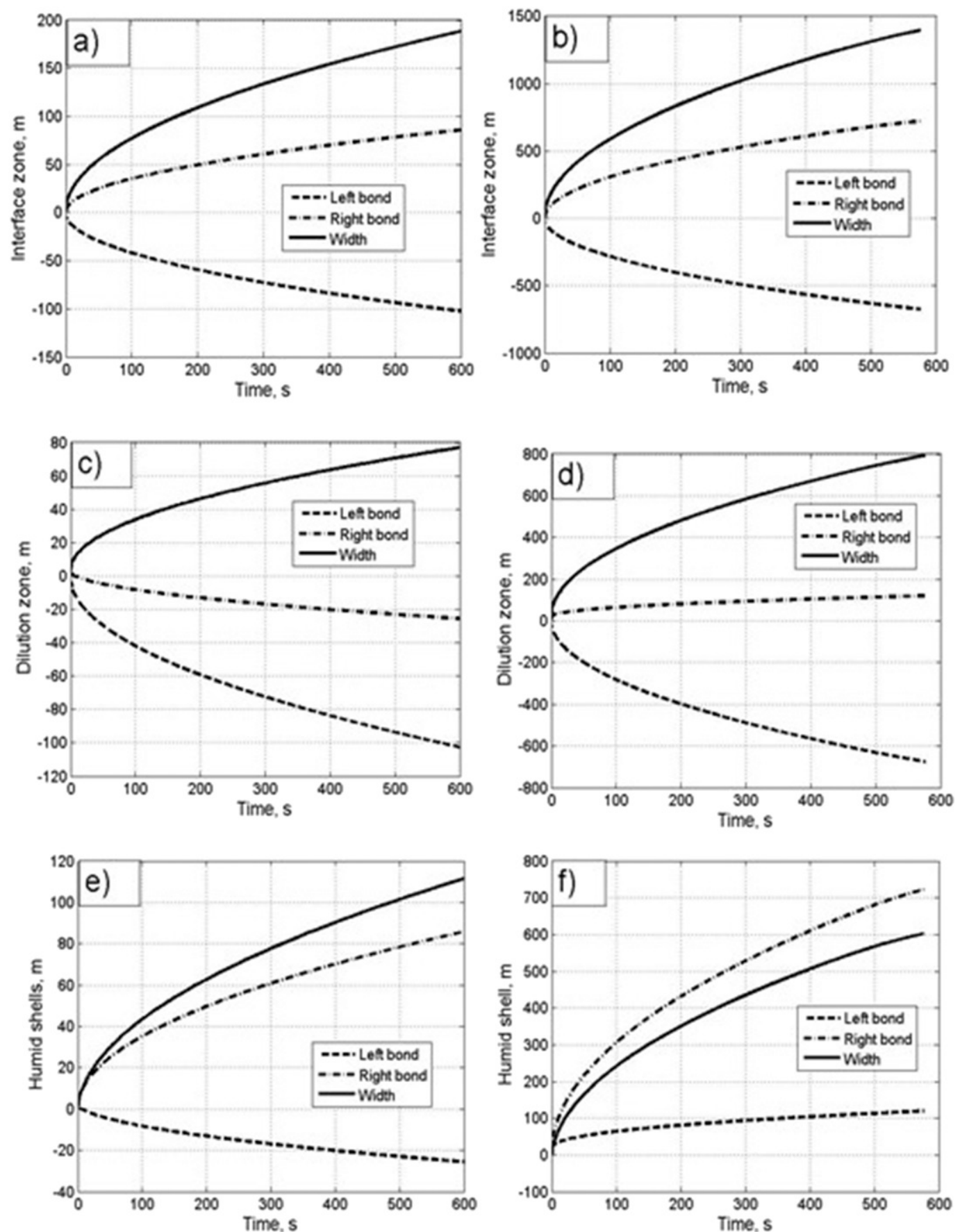

FIG. 10. Time dependence of boundary locations and widths of (a),(b) the interface zone, (c),(d) the cloud dilution zone, and (e),(f) the humid shell for (left) cloud 1 (small $\mathrm{Cu}$ ) and (right) cloud 2 (deep convective).

dilution zone moves outward, toward the dry environment while the left boundary is shifted in the opposite direction. It means that a deep convective cloud expands during entrainment-mixing. However, the internal boundary of the dilution zone shifts toward the cloud interior by about $700 \mathrm{~m}$ over $10 \mathrm{~min}$, while the outer cloud edge shifts outside by only $150 \mathrm{~m}$. If a deep convective cloud width exceeds a few kilometers, its cores are likely to remain close to the adiabatic.

Figure 10e shows that the internal (left) boundary of the humid shell in small $\mathrm{Cu}$ shifts toward the cloud interior, indicating cloud dissipation by evaporation of 


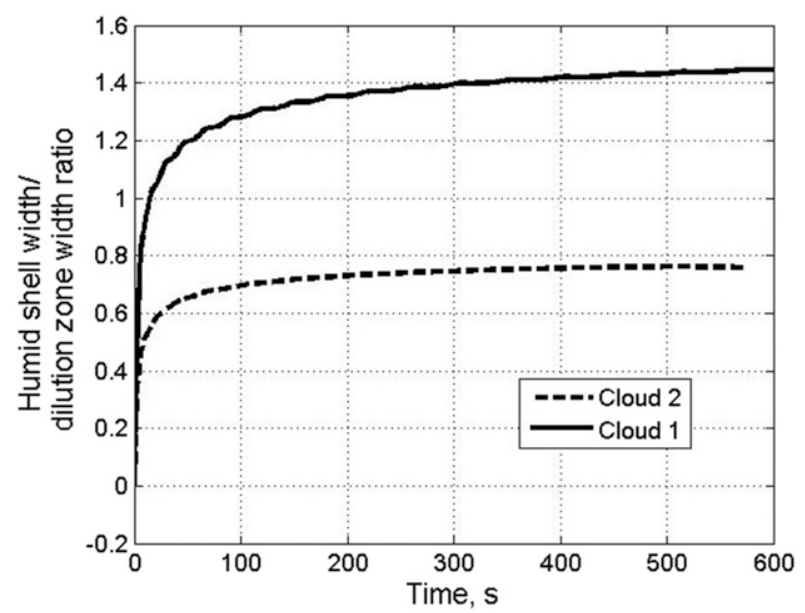

FIG. 11. Dependence of the humid shell/the dilution zone width ratio on time for cloud 1 and cloud 2.

droplets at the cloud edge. The boundaries of the humid shell in $\mathrm{Cu}$ move in opposite directions, which determines a significant expansion of the humid zone. In a deep convective cloud, both boundaries of the humid zone move outside (Fig. 10f).

Figure 11 shows the dependence of the ratio of the humid shell/the dilution zone widths on time for cloud 1 and cloud 2. Since the ratio of the widths is the same in normalized and nonnormalized units, the curves in Fig. 11 are the same as in Fig. 8 but drawn for $R$ values in cloud 1 and cloud 2. One can see that in small $\mathrm{Cu}$ the humid shell is $40 \%$ wider than the dilution zone. Therefore, an evaporating cloud creates a large zone of humid air. In the deep convective cloud, the humid shell is narrower than its dilution zone.

Figure 12 shows spatial dependences of LWC, supersaturation, and the effective radius at $t=2.5 \mathrm{~min}$ within the interface zone for a small $\mathrm{Cu}$ (cloud 1) and a deep convective cloud (cloud 2). Black circles show the boundaries of the cloud dilution zone and the humid shell. In Figs. 12e and 12f, the circles show the boundaries of the dilution zone. Figure 12 allows us to do the following important quantitative estimations related to the dilution zone and the humid shell in small and large $\mathrm{Cu}$ :

- LWC decreases within the dilution zone from its maximum in the cloud interior to a negligible value at the cloud edge within a 60-m segment of the dilution zone of small $\mathrm{Cu}$ (Fig. 12a) and a 300-m segment of deep convective cloud (Fig. 12b).

- Supersaturation remains equal to zero $(\mathrm{RH}=100 \%)$ within over $85 \%$ of the dilution zone width and falls by $2 \%-5 \%$ in the close vicinity of the cloud edge (Figs. 12c,d).
- The effective radius in small $\mathrm{Cu}$ is nearly constant (the decrease is by only $0.5 \mu \mathrm{m}$ ) within a $50-\mathrm{m}$ segment of the dilution zone and rapidly decreases from about 7.5 to $5.5 \mu \mathrm{m}$ (i.e., by $25 \%$ ) within $10 \mathrm{~m}$ in the vicinity of the cloud edge (black point at $x=-22 \mathrm{~m}$ in Fig. 12e).

- In the deep convective cloud (Fig. 12f), the effective radius remains nearly constant within $300 \mathrm{~m}$ of the dilution zone, and decreases from 9.5 to $7 \mu \mathrm{m}$ within about $60 \mathrm{~m}$ near the cloud edge.

We tried to estimate the aerosol effects on characteristics of zones emerging as a result of the entrainmentmixing process. It is known that an increase in aerosol concentration causes increasing droplet concentration and decreasing droplet size. According to the first indirect effect (Twomey 1974, 1977), an increase in aerosol concentration at constant LWC increases the surface of droplets and cloud albedo. To check possible effects of aerosols on the widths of the interface and of the dilution zones, as well as on the width of the humid shell, we halved the droplet concentration in clouds 1 and 2 . The droplet radii were increased to keep the values of $R$ unchanged. As a result, the LWC remained unchanged as well. It was found that these changes do not affect any dependence shown in Fig. 10. The coincidence of the boundaries and the widths of the interface zones follows from Eqs. (13), (15), and (17). Coincidence of the location of the boundaries between the dilution zone and the humid shell might be explained by the fact that the ratio between the widths of the humid shell and of the dilution zone is independent from spatial normalization. Besides, the characteristic relaxation times for both cloud 1 and cloud 2 are much lower than that of turbulent diffusion.

In general, the results of the study show that the effects of aerosols on the sizes of the interface zone and different subzones are likely to be manifested via the effects of aerosols on $R$ values. For instance, an increase in aerosol concentration may affect the LWC value. Of course, a decrease in the initial droplet concentration in the cloud interior leads to a proportional decrease in droplet concentration at all distances within the dilution zone.

\section{Discussion and conclusions}

The entrainment-mixing process leads to formation of an interface cloud-environment zone, consisting of a cloud dilution zone where liquid water content decreases with increasing distance from cloud interior and of a humid shell where the air humidity exceeds that of the dry environment. The location of the 

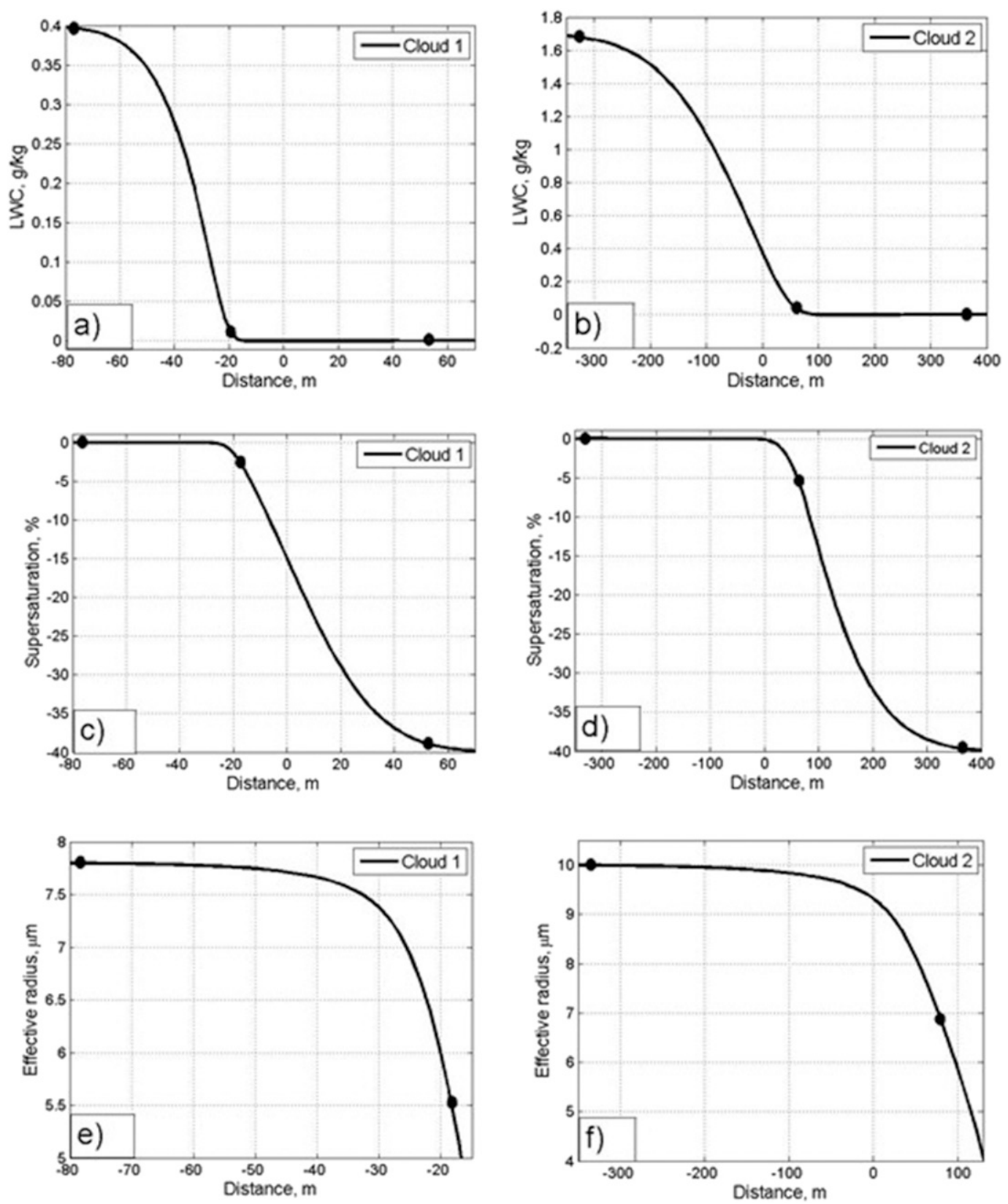

FIG. 12. Dependences of (a),(b) LWC, (c),(d) supersaturation, and (e),(f) the effective radius at $t=2.5$ min within the interface zone for (left) cloud 1 and (right) cloud 2. The black circles in (a)-(d) show boundaries of the cloud dilution zone and the humid shell. In (e) and (f) the black circles show the boundaries of the dilution zone. The distances are measured from the initial cloud edge.

boundaries and of the widths of these zones and subzones changes over time. This time evolution is analyzed by solving the diffusion-evaporation equation for an open region in the vicinity of the cloud-dry air interface. Upon normalization, the problem is reduced to a one-parametric one with the governing parameter being the potential evaporation parameter $R<0$, which is proportional to the ratio of saturation deficit in the dry air to the available liquid water content in the cloud air.

Calculations carried out using the normalized Eqs. (2)-(9) show that widths of the cloud dilution zone and of the humid shell increase with time. At $R<-1$, the interface between the dilution zone and the humid shell (i.e., the cloud edge) moves toward the cloud interior; that is, the cloud dissipates and shrinks because 
of droplet evaporation near the cloud edge. At $R>-1$, the cloud edge moves outward; that is, the mixing of the cloud and the surrounding air leads to an increase in the cloud volume. At $R=-1$, the location of the cloud edge quickly stabilizes and a peculiar balance between cloud and environment takes place.

Parameter $R$ increases with decreasing temperature, since so does coefficient $A_{2}$ [see Eq. (1)]. Within the temperature range from $20^{\circ}$ to $-20^{\circ} \mathrm{C}, A_{2}$ increases by an order of magnitude (Khain and Pinsky 2018, their Fig. 5.2.1). Since temperature decreases with height, clouds at high levels are characterized by larger $R$ and should tend to expand. The physical reason for this conclusion is that saturating water vapor pressure decreases with decreasing temperature. It means that smaller amount of liquid water (lower LWC) is required to eliminate saturation deficit. One can hypothesize that this effect may facilitate the growth of cloud anvils. It might also explain why the lower part of a cloud dissipates faster than its upper part, which is a widely observed phenomenon. One can suppose, therefore, that in most cases, clouds developing in a cold environment (at $T<-10^{\circ} \mathrm{C}$ ) expand, whereas at warmer temperatures, clouds may both shrink or expand depending on the ratio $S_{2} / A_{2}(T)$.

We consider two examples of real clouds: small $\mathrm{Cu}$ and deep convective clouds with parameters obtained in high-frequency in situ measurements by Katzwinkel et al. (2014) and Kumar et al. (2017), respectively. It is shown that the width of the dilution zone for small dissipating $\mathrm{Cu}$ is of several tens meters, while in a growing deep convective cloud, it is of several hundred meters. The widths of the dilution zone in $\mathrm{Cu}$ and in deep convective clouds obtained in the simulations agree well with those obtained in in situ measurements.

The humid shell around clouds forms a little later than the cloud dilution zone, and gradually, its width becomes of the same order as that of the dilution zone. The ratio of the width of the humid shell to the width of dilution zone is about 0.8 for a growing cloud and may reach 1.4 for dissipating $\mathrm{Cu}$. The existence of a significant humid shell around clouds was established in several studies (Gerber et al. 2008; Lehmann et al. 2009; Bar-Or et al. 2012) in relation to different problems of cloud microphysics and cloud radiation properties. In multiple studies dedicated to problems of mixing of clouds with surrounding air, it is assumed that the volumes of dry air with characteristic sizes of several tens of meters penetrate clouds and then mix up with the cloud interior air (e.g., Baker et al. 1980; Baker and Latham 1982; Raga et al. 1990; Krueger et al. 1997; Su et al. 1998). In these studies, the processes of entrainment and mixing were considered sequentially. The results of such mixing substantially depend on RH of the penetrating air, which is typically assumed equal to that of the dry environment. However, the humid shell was not taken into account, assuming a sharp boundary between the cloudy air and the dry environment.

In the present model, we view the processes of entrainment and mixing as a single entrainment-mixing process leading to a rapid formation of the interface zone instead of the sharp gradients. In this process, a cloud is surrounded by the humid shell of several tens to hundred meters in width. According to our diffusionevaporation model, the humidity of the air penetrating the cloud (the humidity at cloud boundary) exceeds $90 \%$ (Figs. 12c,d). RH in the dry environment determines the widths of the dilution zone and of the humid shell and the regime (growth versus evaporation) of cloud evolution.

It is of interest that the dilution zone grows largely in the direction of the cloud interior regardless of the value of $R$. At the same time, the effective radius remains nearly constant within a larger part of the dilution zone, its values being close to those in the cloud interior. This behavior of the effective radius explains its low variability per level even within clouds containing wide dilution zones. In in situ observations, the zone of decreasing effective radius in the close vicinity of the cloud edge can be found only by means of highfrequency measurements allowing resolution of $10 \mathrm{~m}$, as in the study by Kumar et al. (2017). This property of the effective radius makes its vertical profile in clouds robust, so the cloud may be characterized entirely by a single profile of the effective radius. (Freud et al. 2008, 2011; Rosenfeld et al. 2014; Khain et al. 2018).

The estimations of propagation velocity of the dilution zone toward the cloud interior show that clouds wider than several hundred meters may contain interiors consisting of slightly diluted volumes only during several tens of minutes. Small $\mathrm{Cu}$ of a few hundred meters in size become diluted within $10-15 \mathrm{~min}$. It is quite likely that cloud dilution affects the lifetime of clouds, especially of small $\mathrm{Cu}$.

Additional calculations were performed for the parameters of the interface zone as well of the subzones of the analogous small $\mathrm{Cu}$ and deep convective clouds, but with halved droplet concentration. To keep $R$ values unchanged, the initial droplet sizes were increased accordingly. These changes in droplet concentration and droplet size simulate a situation of decreasing aerosol concentration. It was shown that aerosols can affect the widths of the interface zone, the dilution zone, and the humid shell only via changes of $R$ values, that is, via the effects on cloud LWC. 
The entrainment-mixing process is a complicated phenomenon that includes many dynamical and microphysical aspects. It is clear that the mixing-evaporation equation describes this process only approximately, as it treats the entrainment-mixing as a process of turbulent diffusion characterized by a certain turbulent coefficient. As a result, our model being based on this equation predicts smoothed monotonic profiles of all the quantities within the interface zone. This does not describe the evolution of separate turbulent filaments, which introduces fluctuations into the profiles of LWC and of droplet number concentration. Strictly speaking, our approach is valid when turbulent vortices entraining clouds are much smaller than the width of the interface zone and of the subzones. Analysis of experimental data (Gerber et al. 2008; Bera et al. 2016a) shows that this assumption is reasonable. Besides, the proposed model assumes no vertical motions at the cloud edge, which can be another substantial limitation, especially when evaluating cloud lifetime. We believe that the model assumptions are more suitable for both developing and mature clouds. At the stage of dissipation, downdrafts can be a more substantial factor of cloud dissipation than mixing with the environment.

Nevertheless, the model shows good physically grounded results and can reproduce many observable features of clouds near the cloud edge. We believe that the method and the results of the study may be applied for analysis and interpretation of remote sensing optical data obtained near cloud edges (Marshak and Yang 2018; Grosvenor et al. 2018). It can also prove useful for investigating the effects of mixing on cloud microphysics.

Acknowledgments. This research was supported by the Israel Science Foundation (Grants 1393/14 and 2027/ 17). Partial support for this work comes from Grants DE-SC008811, DE-SC0014295, and ASR DE-FOA-1638 from the U.S. Department of Energy Atmospheric System Research program.

\section{APPENDIX A}

\section{Derivation of Diffusion-Evaporation Equations}

\section{a. Conservative quantity $\Gamma$}

The equation for water vapor supersaturation in an adiabatic air volume can be written in the form (e.g., Pruppacher and Klett 1997; Pinsky et al. 2013)

$$
\frac{d S}{d t}=A_{1} \frac{d z}{d t}-A_{2} \frac{d q}{d t}
$$

where $S$ is supersaturation, $q$ is the liquid water mixing ratio, $d z / d t$ is the vertical velocity of the air volume, and coefficients $A_{1}$ and $A_{2}$ slightly depend on temperature (see appendix B). Assuming coefficients $A_{1}$ and $A_{2}$ are constant, which means neglecting the temperature changes during droplet condensation/ evaporation, the integration of Eq. (A1) leads to a balance equation:

$$
S=A_{1} z-A_{2} q+C,
$$

where $z$ is the vertical coordinate and $C=$ const is determined by the initial conditions. Equations (A1) and (A2) are valid in case $|S| \ll 1$. If the air volume does not move in the vertical direction $(z=$ const $)$, the quantity

$$
\Gamma=S+A_{2} q
$$

is invariant with respect to moist adiabatic processes; that is, one can assume that $\Gamma$ is a conservative value.

The entrainment-detrainment-mixing of conservative quantity $\Gamma$ in the horizontal direction is described by the following nonstationary equation of turbulent diffusion:

$$
\frac{\partial \Gamma(x, t)}{\partial t}=K \frac{\partial^{2} \Gamma(x, t)}{\partial x^{2}}
$$

where $K$ is the coefficient of turbulent diffusion. Equation (A4) is solved with the initial condition (see Fig. 1)

$$
\Gamma(x, 0)=\left\{\begin{array}{lll}
\Gamma_{1}=A_{2} q_{1} & \text { if } & x \leq 0 \\
\Gamma_{2}=S_{2} & \text { if } & x>0
\end{array}\right.
$$

using the Dirichlet boundary conditions at $x= \pm \infty$ :

$$
\Gamma(-\infty, t)=\Gamma_{1} ; \quad \Gamma(\infty, t)=\Gamma_{2} .
$$

The nonstationary solution of Eq. (A4) with the initial and boundary conditions (A5) and (A6) is represented by the error function (erf; Polyanin and Zaitsev 2004)

$$
\Gamma(x, t)=\frac{1}{2}\left[\left(\Gamma_{1}+\Gamma_{2}\right)-\left(\Gamma_{1}-\Gamma_{2}\right) \operatorname{erf} \frac{x}{2 \sqrt{K t}}\right] .
$$

The normalized solution $\tilde{\Gamma}(x, t)=\Gamma(x, t) /\left(A_{2} q_{1}\right)=$ $\Gamma(x, t) / \Gamma_{1}$ depends on two parameters, namely, the turbulent coefficient $K$ and the PEP $R$, introduced in Pinsky et al. (2016b):

$$
R=\frac{S_{2}}{A_{2} q_{1}}=\frac{\Gamma_{2}}{\Gamma_{1}} .
$$


Using Eq. (A8), the normalized solution can be written as

$$
\tilde{\Gamma}(x, t)=\frac{1}{2}\left[(1+R)-(1-R) \operatorname{erf} \frac{x}{2 \sqrt{K t}}\right] .
$$

\section{b. Equation for droplet size distribution}

We define distribution of droplets with respect to their square radii $\sigma=r^{2}, g(x, t, \sigma)$, which obeys the normalization condition

$$
N(x, t)=\int_{0}^{\infty} g(x, t, \sigma) d \sigma,
$$

where $N(x, t)$ is droplet number concentration. In cloud physics, the DSD $f(x, t, r)$ that has the normalization $N(x, t)=\int_{0}^{\infty} f(x, t, r) d r$ is usually used. The distribution $g(x, t, \sigma)$ relates to $f(x, t, r)$ as $f(x, t, r)=2 \operatorname{rg}(x, t, \sigma)$. Using $g(x, t, \sigma)$, the liquid water mixing ratio can be represented by integral

$$
q(x, t)=\frac{4 \pi \rho_{w}}{3 \rho_{a}} \int_{0}^{\infty} \sigma^{3 / 2} g(\sigma, x, t) d \sigma .
$$

The evaporation of cloud droplets is described by (Pruppacher and Klett 1997)

$$
\frac{d \sigma}{d t}=\frac{2 S}{F},
$$

where coefficient $F$ slightly depends on temperature and pressure (see appendix B). We assume that $F$ does not change in the course of mixing.

The 1D diffusion-evaporation equation for nonconservative function $g(\sigma)$ can be written in the form suggested by Rogers and Yau (1996; see also Pinsky et al. 2016b):

$$
\frac{\partial g(\sigma)}{\partial t}=K \frac{\partial^{2} g(\sigma)}{\partial x^{2}}-\frac{\partial}{\partial \sigma}\left[\frac{d \sigma}{d t} g(\sigma)\right] .
$$

The substitution of Eq. (A12) into Eq. (A13) leads to a diffusion-evaporation equation written in the form obtained by Pinsky et al. (2016b):

$\frac{\partial g(x, t, \sigma)}{\partial t}=K \frac{\partial^{2} g(x, t, \sigma)}{\partial x^{2}}-\frac{2 S(x, t)}{F} \frac{\partial g(x, t, \sigma)}{\partial \sigma}$,

in which supersaturation $S(x, t)$ is calculated from Eq. (A3).

The diffusion-evaporation equation is solved for the case of monodisperse initial DSD inside a cloud. This initial DSD is represented as a step function (see Fig. 1):

$$
g(x, 0, \sigma)=\left\{\begin{array}{ccc}
N_{1} \delta\left(\sigma-\sigma_{1}\right) & \text { if } & x \leq 0 \\
0 & \text { if } & x>0
\end{array},\right.
$$

where $N_{1}$ is droplet concentration inside the cloud, $\sigma_{1}=r_{1}^{2}$ is the square of the initial cloud droplet radius, and $\delta$ is the delta function. Equation (A14) should be solved with the Dirichlet boundary conditions (see Fig. 1)

$$
g(-\infty, t, \sigma)=N_{1} \delta\left(\sigma-\sigma_{1}\right) ; \quad g(\infty, t, \sigma)=0 .
$$

Since the boundaries are very remote from the physical edge of the cloud, the gradients of all the variables are actually zero at the boundaries.

Equations (A3), (A11), (A14), and (A7) form a closed equation system.

\section{c. Nondimensional form of diffusion-evaporation equation}

To reduce the number of variables that affect the solution of Eq. (A14), we define several nondimensional variables (see Table 1; to normalize the time variable, we use the initial phase relaxation time inside the undisturbed cloud)

$$
\tau_{1}=\frac{\rho_{a} F}{4 \pi \rho_{w} A_{2} N_{1} r_{1}}
$$

Instead of Eqs. (A14), (A11), and (A7), we use the corresponding nondimensional equations:

$$
\begin{aligned}
\frac{\partial \tilde{g}(\tilde{x}, \tilde{t}, \tilde{\sigma})}{\partial \tilde{t}} & =\frac{\partial^{2} \tilde{g}(\tilde{x}, \tilde{t}, \tilde{\sigma})}{\partial \tilde{x}^{2}}+\frac{2}{3}[\tilde{\Gamma}(\tilde{x}, \tilde{t})-\tilde{q}(\tilde{x}, \tilde{t})] \frac{\partial \tilde{g}(\tilde{x}, \tilde{t}, \tilde{\sigma})}{\partial \tilde{\sigma}}, \\
\tilde{q}(\tilde{x}, \tilde{t}) & =\int_{0}^{\infty} \tilde{\sigma}^{3 / 2} \tilde{g}(\tilde{x}, \tilde{t}, \tilde{\sigma}) d \tilde{\sigma}, \\
\tilde{\Gamma}(\tilde{x}, \tilde{t}) & =\frac{1}{2}\left[(1+R)-(1-R) \operatorname{erf} \frac{\tilde{x}}{2 \sqrt{\tilde{t}}}\right] .
\end{aligned}
$$

Equation (A18) should be solved under the normalized initial condition

$$
\tilde{g}(\tilde{x}, 0, \tilde{\sigma})=\left\{\begin{array}{ccc}
\delta(\tilde{\sigma}-1) & \text { if } & \tilde{x} \leq 0 \\
0 & \text { if } & \tilde{x}>0
\end{array}\right.
$$

and normalized boundary conditions

$$
\tilde{g}(-\infty, \tilde{t}, \tilde{\sigma})=\delta(\tilde{\sigma}-1) ; \quad \tilde{g}(\infty, \tilde{t}, \tilde{\sigma})=0 .
$$

Since $\tilde{g}(\tilde{x}, \tilde{t}, \tilde{\sigma})$ is the solution of Eqs. (A18)-(A20) containing the sole nondimensional parameter $R$, it depends solely on $R$ defined in Eq. (A8). 
TABLE B1. List of symbols.

\begin{tabular}{|c|c|c|}
\hline Symbol & Description & Units \\
\hline$A_{1}$ & $\frac{g}{R_{a} T}\left(\frac{L_{w} R_{a}}{c_{p} R_{v} T}-1\right)$, coefficient & $\mathrm{m}^{-1}$ \\
\hline$A_{2}$ & $\frac{1}{q_{v}}+\frac{L_{w}^{2}}{c_{p} R_{v} T^{2}}$, coefficient & - \\
\hline$c_{p}$ & Specific heat capacity of moist air at constant pressure & $\mathrm{J} \mathrm{kg}^{-1} \mathrm{~K}^{-1}$ \\
\hline $\mathscr{D}$ & Coefficient of water vapor diffusion in the air & $\mathrm{m}^{2} \mathrm{~s}^{-1}$ \\
\hline$e$ & Water vapor pressure & $\mathrm{N} \mathrm{m}^{-2}$ \\
\hline$e_{S}$ & Saturation vapor pressure above a flat water surface & $\mathrm{Nm}^{-2}$ \\
\hline$F$ & $F=\frac{\rho_{w} L_{w}^{2}}{k R T^{2}}+\frac{\rho_{w} R_{v} T}{\rho_{c}(T) \mathscr{C}}$, coefficient & $\mathrm{m}^{-2} \mathrm{~s}$ \\
\hline$g(\sigma)$ & $\begin{array}{c}K_{a} R_{v} I^{2} \quad e_{S}(T) \\
\text { Distribution of square radius }\end{array}$ & $\mathrm{m}^{-5}$ \\
\hline$\tilde{g}(\tilde{\sigma})$ & Normalized distribution of square radius & - \\
\hline $\begin{array}{l}g(0) \\
k_{a}\end{array}$ & Coefficient of air heat conductivity & $\mathrm{J} \mathrm{m}^{-1} \mathrm{~s}^{-1} \mathrm{~K}^{-1}$ \\
\hline$K$ & Turbulent diffusion coefficient & $\mathrm{m}^{2} \mathrm{~s}^{-1}$ \\
\hline$L_{w}$ & Latent heat for liquid water & $\mathrm{J} \mathrm{kg}^{-1}$ \\
\hline$N$ & Droplet concentration & - \\
\hline$\tilde{N}$ & Normalized droplet concentration & - \\
\hline$N_{1}$ & Initial droplet concentration in a cloud & $\mathrm{m}^{-3}$ \\
\hline$p$ & Pressure of moist air & $\mathrm{Nm}^{-2}$ \\
\hline$q$ & Liquid water mixing ratio & $\mathrm{kg} \mathrm{kg}^{-1}$ \\
\hline$q_{1}$ & Initial liquid water mixing ratio in a cloud & $\mathrm{kg} \mathrm{kg}^{-1}$ \\
\hline$q_{v}$ & Water vapor mixing ratio & $\mathrm{kg} \mathrm{kg}^{-1}$ \\
\hline$\tilde{q}$ & Normalized liquid water mixing ratio (equal to normalized LWC) & - \\
\hline$r$ & Droplet radius & $\mathrm{m}$ \\
\hline$r_{1}$ & Initial droplet radius & $\mathrm{m}$ \\
\hline$R$ & $\frac{S_{2}}{}$, potential evaporation parameter & - \\
\hline$R_{a}$ & Specific gas constant of moist air & $\mathrm{J} \mathrm{kg}^{-1} \mathrm{~K}^{-1}$ \\
\hline$R_{v}$ & Specific gas constant of water vapor & $\mathrm{J} \mathrm{kg}^{-1} \mathrm{~K}^{-1}$ \\
\hline$S$ & $e / e_{w}-1$, supersaturation over water & - \\
\hline$\tilde{S}$ & Normalized supersaturation & - \\
\hline$S_{2}$ & Initial supersaturation in dry air & - \\
\hline$T$ & Temperature & $\mathrm{K}$ \\
\hline$t$ & Time & $\mathrm{s}$ \\
\hline$\tilde{t}$ & Nondimensional time & - \\
\hline$x$ & Distance & $\mathrm{m}$ \\
\hline$\tilde{x}$ & Nondimensional distance & - \\
\hline$\Gamma(x, t)$ & Conservative function & - \\
\hline$\tilde{\Gamma}$ & Normalized conservative function & - \\
\hline$\rho_{a}$ & Air density & $\mathrm{kg} \mathrm{m}^{-3}$ \\
\hline$\rho_{w}$ & Density of liquid water & $\mathrm{kg} \mathrm{m}^{-3}$ \\
\hline$\sigma w$ & Square of droplet radius & $\mathrm{m}^{2}$ \\
\hline$\tilde{\sigma}$ & Normalized square of droplet radius & - \\
\hline$\tau_{1}$ & Initial phase relaxation time & $\mathrm{s}$ \\
\hline
\end{tabular}

\section{APPENDIX B}

\section{List of Symbols}

Symbols used in this paper are listed in Table B1

\section{REFERENCES}

Baker, M. B., and J. Latham, 1982: A diffusive model of the turbulent mixing of dry and cloudy air. Quart. J. Roy. Meteor. Soc., 108, 871-898, https://doi.org/10.1002/qj.49710845809.

—, R. G. Corbin, and J. Latham, 1980: The influence of entrainment on the evolution of cloud drop spectra: I. A model of inhomogeneous mixing. Quart. J. Roy. Meteor. Soc., 106, 581-598, https://doi.org/10.1002/qj.49710644914.

Bar-Or, R. Z., I. Koren, O. Altaratz, and E. Fredj, 2012: Radiative properties of humidified aerosol in cloudy environment. Atmos. Res., 118, 280-294, https://doi.org/10.1016/ j.atmosres.2012.07.014.

Bera, S., T. V. Prabha, and W. W. Grabowski, 2016a: Observations of monsoon convective cloud microphysics over India and role of entrainment-mixing. J. Geophys. Res. Atmos., 121, 97679788, https://doi.org/10.1002/2016JD025133.

, G. Pandithurai, and T. V. Prabha, 2016b: Entrainment and droplet spectral characteristics in convective clouds during transition to monsoon. Atmos. Sci. Lett., 17, 286-293, https:// doi.org/10.1002/asl.657. 
Freud, E., D. Rosenfeld, M. O. Andreae, A. A. Costa, and P. Artaxo, 2008: Robust relations between CCN and the vertical evolution of cloud drop size distribution in deep convective clouds. Atmos. Chem. Phys., 8, 1661-1675, https:// doi.org/10.5194/acp-8-1661-2008.

,-- , and J. R. Kulkarni, 2011: Resolving both entrainmentmixing and number of activated $\mathrm{CCN}$ in deep convective clouds. Atmos. Chem. Phys., 11, 12 887-12 900, https://doi.org/ 10.5194/acp-11-12887-2011.

Gerber, H., 2000: Structure of small cumulus clouds. Proc. 13th Int. Conf. on Clouds and Precipitation, Reno, NV, WMO, 105-108.

—_, G. Frick, J. B. Jensen, and J. G. Hudson, 2008: Entrainment, mixing, and microphysics in trade-wind cumulus. J. Meteor. Soc. Japan, 86A, 87-106, https://doi.org/10.2151/ jmsj.86A.87.

Grosvenor, D., and Coauthors, 2018: Remote sensing of cloud droplet number concentration in warm clouds: Review of current state of knowledge and perspectives. Rev. Geophys., 56, 409-453, https://doi.org/10.1029/2017RG000593.

Heus, T., and H. J. J. Jonker, 2008: Subsiding shells around shallow cumulus clouds. J. Atmos. Sci., 65, 1003-1018, https://doi.org/ 10.1175/2007JAS2322.1.

Katzwinkel, J., H. Siebert, T. Heus, and R. A. Shaw, 2014: Measurements of turbulent mixing and subsiding shells in trade wind cumuli. J. Atmos. Sci., 71, 2810-2822, https://doi.org/ 10.1175/JAS-D-13-0222.1.

Khain, A. P., and M. Pinsky, 2018: Physical Processes in Clouds and Cloud Modelling. Cambridge University Press, $626 \mathrm{pp}$.

,-- , and L. Magaritz-Ronen, 2018: Physical interpretation of mixing diagrams. J. Geophys. Res. Atmos., 123, 529-542, https://doi.org/10.1002/2017JD027124.

Khain, P., and Coauthors, 2019: Parameterization of vertical profiles of governing microphysical parameters of shallow cumulus cloud ensembles using LES with bin microphysics. J. Atmos. Sci., 76, 533-560, https://doi.org/10.1175/JAS-D-18-0046.1.

Krueger, S., C.-W. Su, and P. McMurtry, 1997: Modeling entrainment and finescale mixing in cumulus clouds. J. Atmos. Sci., 54, 2697-2712, https://doi.org/10.1175/1520-0469(1997)054<2697: MEAFMI $>2.0 . \mathrm{CO} ; 2$.

Kumar, B., S. Bera, T. V. Prabha, and W. W. Grabowski, 2017: Cloud-edge mixing: Direct numerical simulation and observations in Indian monsoon clouds. J. Adv. Model. Earth Syst., 9, 332-353, https://doi.org/10.1002/2016MS000731.

Lehmann, K., H. Siebert, and R. A. Shaw, 2009: Homogeneous and inhomogeneous mixing in cumulus clouds: Dependence on local turbulence structure. J. Atmos. Sci., 66, 3641-3659, https://doi.org/10.1175/2009JAS3012.1.

Magaritz-Ronen, L., M. Pinsky, and A. Khain, 2016: Drizzle formation in stratocumulus clouds: Effects of turbulent mixing. Atmos. Chem. Phys., 16, 1849-1862, https://doi.org/10.5194/ acp-16-1849-2016.

Marshak, A., and W. Yang, 2018: Study of cloud-aerosol interactions and mixing processes in the clear-to-cloud transition zone using ARM shortwave spectrometers. Joint ARM User Facility and ASR PI Meeting, Tysons, VA, Atmospheric System Research, https://asr.arm.gov/meetings/stm/posters/ view?id=1982.
Monin, A. S., and A. M. Yaglom, 1975: Statistical Fluid Mechanics. Mechanics of Turbulence. Vol. 2. MIT Press, $886 \mathrm{pp}$.

Park, S.-B., T. Heus, and P. Gentine, 2017: Role of convective mixing and evaporative cooling in shallow convection. J. Geophys. Res. Atmos., 122, 5351-5363, https://doi.org/10.1002/ 2017JD026466.

Pinsky, M., and A. Khain, 2018: Theoretical analysis of the entrainmentmixing process at cloud boundaries. Part I: Droplet size distributions and humidity within the interface zone. J. Atmos. Sci., 75, 2049-2064, https://doi.org/10.1175/JAS-D-17-0308.1.

_ I. P. Mazin, A. Korolev, and A. Khain, 2013: Supersaturation and diffusional droplet growth in liquid clouds. J. Atmos. Sci., 70, 2778-2793, https://doi.org/10.1175/JAS-D-12-077.1.

_- A. Khain, A. Korolev, and L. Magaritz-Ronen, 2016a: Theoretical investigation of mixing in warm clouds-Part 2: Homogeneous mixing. Atmos. Chem. Phys., 16, 9255-9272, https://doi.org/10.5194/acp-16-9255-2016.

,$- \ldots$, and $-2016 \mathrm{~b}$ : Theoretical analysis of mixing in liquid clouds-Part 3: Inhomogeneous mixing. Atmos. Chem. Phys., 16, 9273-9297, https://doi.org/10.5194/acp-16-9273-2016.

Plant, R. S., and J.-I. Yano, 2015: Theoretical Background and Formulation. Vol. 1, Parameterization of Atmospheric Convection, Imperial College Press, 515 pp.

Polyanin, A. D., and V. F. Zaitsev, 2004: Handbook of Nonlinear Partial Differential Equations. Chapman and Hall, 809 pp.

Pruppacher, H. R., and J. D. Klett, 1997: Microphysics of Clouds and Precipitation. 2nd ed. Oxford Press, 914 pp.

Raga, G. B., J. B. Jensen, and M. B. Baker, 1990: Characteristics of cumulus band clouds off the coast of Hawaii. J. Atmos. Sci., 47, 338-355, https://doi.org/10.1175/1520-0469(1990)047<0338: COCBCO $>2.0 . \mathrm{CO} ; 2$.

Rodts, S. M. A., P. G. Duynkerke, and H. J. J. Jonker, 2003: Size distributions and dynamical properties of shallow cumulus clouds from aircraft observations and satellite data. J. Atmos. Sci., 60, 1895-1912, https://doi.org/10.1175/1520-0469(2003) 060<1895:SDADPO $>2.0 . C O ; 2$.

Rogers, R. R., and M. K. Yau, 1996: Short Course in Cloud Physics. Butterworth-Heinemann, 304 pp.

Rosenfeld, D., B. Fischman, Y. Zheng, T. Goren, and D. Giguzin, 2014: Combined satellite and radar retrievals of drop concentration and $\mathrm{CCN}$ at convective cloud base. Geophys. Res. Lett., 41, 3259-3265, https://doi.org/10.1002/2014GL059453.

Schmeissner, T., R. A. Shaw, J. Ditas, F. Stratmann, M. Wendisch, and H. Siebert, 2015: Turbulent mixing in shallow trade wind cumuli: Dependence on cloud life cycle. J. Atmos. Sci., 72, 1447-1465, https://doi.org/10.1175/JAS-D-14-0230.1.

Su, C.-W., S. K. Krueger, P. A. McMurtry, and P. H. Austin, 1998: Linear eddy modeling of droplet spectral evolution during entrainment and mixing in cumulus clouds. Atmos. Res., 4748, 41-58, https://doi.org/10.1016/S0169-8095(98)00039-8.

Twomey, S., 1974: Pollution and the planetary albedo. Atmos. Environ., 8, 1251-1256, https://doi.org/10.1016/0004-6981(74) 90004-3.

_ 1977: The influence of pollution on the shortwave albedo of clouds. J. Atmos. Sci., 34, 1149-1152, https://doi.org/10.1175/ 1520-0469(1977)034<1149:TIOPOT>2.0.CO;2. 